School of Medicine and Surgery

PhD program in Neuroscience

$31^{\text {st }}$ Cycle

\title{
RECENT SUICIDE ATTEMPTS AND SERUM LIPID PROFILE IN SUBJECTS WITH MENTAL DISORDERS: A CROSS-SECTIONAL STUDY
}

PhD student: Dott. Enrico Capuzzi

Registration number: 809291

Tutor: Chiar.mo Prof. Massimo Clerici

Coordinator: Chiar.mo Prof. Guido Cavaletti

ACADEMIC YEAR: 2017-2018 
To Matilde

To those who told me: Keep moving 


\section{TABLE OF CONTENTS}

ABSTRACT




\section{LIST OF ABBREVATIONS}

$\mathrm{Apo}=$ Apolipoprotein

$\mathrm{ADL}=$ Astrocyte-derived lipoprotein

$\mathrm{BD}=$ Bipolar disorder

$\mathrm{BDNF}=$ Brain-derived neurotrophic factor

$\mathrm{BMI}=$ Body mass index

$\mathrm{CI}=$ Confidence interval

$\mathrm{DF}=$ Degrees of freedom

$\mathrm{FEP}=$ First episode psychosis;

FGA $=$ First generation antipsychotic

$\mathrm{HDL}=$ High density lipoprotein

HMG-CoA=5-Hydroxy-3-methylglutaryl-coenzyme A

HPA=Hypothalamic-pituitary-adrenal

$\mathrm{ICD}=$ International Statistical Classification of Diseases and Related Health Problems

LAI=Long acting injectable antipsychotic

$\mathrm{LDL}=$ Low density lipoprotein

LDL-R $=$ LDL receptor

LRP1=LDL-related protein 1

$\mathrm{MDD}=$ Major depressive disorder

$\mathrm{OR}=\mathrm{Odd}$ ratio

$\mathrm{PD}=$ Personality disorder

PUFAs $=$ Polyunsaturated essential fatty acids

$\mathrm{SCZ}=$ Schizophrenia

$\mathrm{SGA}=$ Second generation antipsychotic

$\mathrm{SMD}=$ Standardized mean difference

VLDL=Very low density lipoprotein

$\mathrm{WMD}=$ Weighted mean difference

$5 \mathrm{HT}=$ Serotonin

5-HTT=Serotonin transporter 


\section{ABSTRACT}

Background: Subjects with mental disorders have a higher risk of suicide behaviors than the general population. So, to date, researchers have investigated some biomarkers possibly related to suicidality. Despite many studies have reported a possible relationship between low lipid serum levels and suicide attempt, conflicting results have emerged.

Aim We investigated whether serum total cholesterol, LDL cholesterol and triglycerides are associated with recent suicide attempts in subjects with different mental disorders.

Methods We conducted a cross-sectional study, including 593 consecutively admitted inpatients with schizophrenia spectrum, bipolar, major depressive, and personality disorders. Serum lipid levels were compared between subjects admitted for a recent suicide attempt and those without such recent history. Moreover, according to hypothesis that links impulsivity and violence with low serum lipid levels, the association between lipid levels and violent suicide attempt was assessed.

Results We did not find any association of total and LDL cholesterol and triglycerides with suicide attempts, also considering diagnosis and suicide methods. In addition, a post-hoc analysis showed a trend toward significance $(\mathrm{p}=0.06)$ in the association between high cholesterol level $(\geq 160 \mathrm{mg} / \mathrm{dL})$ and recent suicide attempt.

Conclusions Our results do not support the hypothesis of association between lipid profiles and suicide attempts in subjects with different mental disorders. Further research is needed to clarify the role of biomarkers in suicidal behaviors. 


\section{CHAPTER 1}

\section{SUICIDE AND SUICIDAL BEHAVIOR}

\subsection{DEFINITION AND RISK FACTORS}

Suicide is a serious public health problem around the world. It's in the top ten most common causes of death in several countries, whilst in younger individuals, aged between 15 and 34, suicide is included in the top three causes of death (Nock et al., 2008). Suicide is defined as the act of intentionally ending of own life while suicidal thoughts and behaviors are classified into three categories: suicide ideation (thoughts of committing behaviors to end own life), suicide plan (formulation of specific method to end own life, without any action) and suicide attempt (selfinjurious behavior of a person willing to die). Suicide attempt, in turn, can be divided on the basis of methods in violent (firearm, hanging, cutting, jumping, car exhaust, other violent methods) and non-violent (drug overdose and poisoning) attempt (Stenbacka and Jokinen, 2015). However suicidal attempt can be distinguished from non suicidal self-injury, in which there is no intention of dying (Nock et al, 2006).

Suicide attempt is considered the most predictive risk factor for a next suicide behavior, in particular during the first year (Suominen et al., 2004). Nevertheless several studies have reported other relevant risk factors, including demographics (female sex, being an adolescent or older adult, being unmarried, unemployed, lower educational attainment) and related to stressful life events (child maltreatment, family conflicts, presence of legal problems, persistent stress in some occupations such as physicians and police officers, being affected by chronic pain or terminal illness) (Nock et al., 2008). However a concurrent psychiatric disorder is the most frequently described risk factor for suicide attempt. Up to $90-95 \%$ of the people committing suicidal behaviors has a psychiatric disorder (Pompili et al., 2005). In particular psychotic, mood, personality and alcohol/substance use, disorders, especially when comorbid, convey the highest risk for suicidal behavior (Chapman et al., 2015). On the other hand specific constructs as hopeless, anhedonia and 
impulsiveness, may increase the risk of suicide attempt as part of mental disorder (Loas et al., 2016).However, suicide attempters with a violent method differ from non-violent attempters. In particular violent attempters are younger, with past conduct problems at school and more predictive of subsequent suicidal behavior than non violent-attempters (Stenbacka and Jokinen, 2015), as well as increased impulsiveness (McMahon et al., 2018). Moreover violent suicide attempters may present some neurobiological differences in comparison to non-violent suicide attempter (Isung et al.,2014).

Here we will focus on suicide attempt behavior because it is linked to suicide more tightly than suicidal ideation in terms of biological markers with potential for predicting risk of suicide (Sudol et al., 2017).

\subsection{BIOMARKERS}

In the last years many researchers have taken an interest on the search of biomarkers as additional tool to prevent suicide. In particular two main categories have been investigated: structural and functional brain imaging and biochemical variables related to HPA axis, neurotransmitters, metabolism of lipids, inflammatory system ("inflammasome") and neuroplasticity (Kim and Lee,2015).

Brain imaging studies report structural and functional abnormalities in the gray and white matter of suicide attempters relative to non attempters (Olie et al., 2015). In magnetic resonance imaging studies suicide attempters showed less gray matter density in temporal and prefrontal cortices and insula. Furthermore some alterations in the white matter volume of frontal and parietal regions, cerebellum, corpus callosum and amygdale have been found in suicide attempters (Jollant et al., 2010). In summary matter abnormalities mainly affect neural networks involved in decision-making and emotional processing. However, despite many published reports, several limitations including small samples, lack of both healthy and psychiatric control groups, different scanning methods across studies, should be considered. Next to imaging studies, there is a growing emphasis on 
neurotransmitter systems, neuroinflammation and molecules involved in neuronal plasticity as possible "actors" in the pathophysiology of suicidal behavior (Sudol et al, 2017; Fig.1 ).

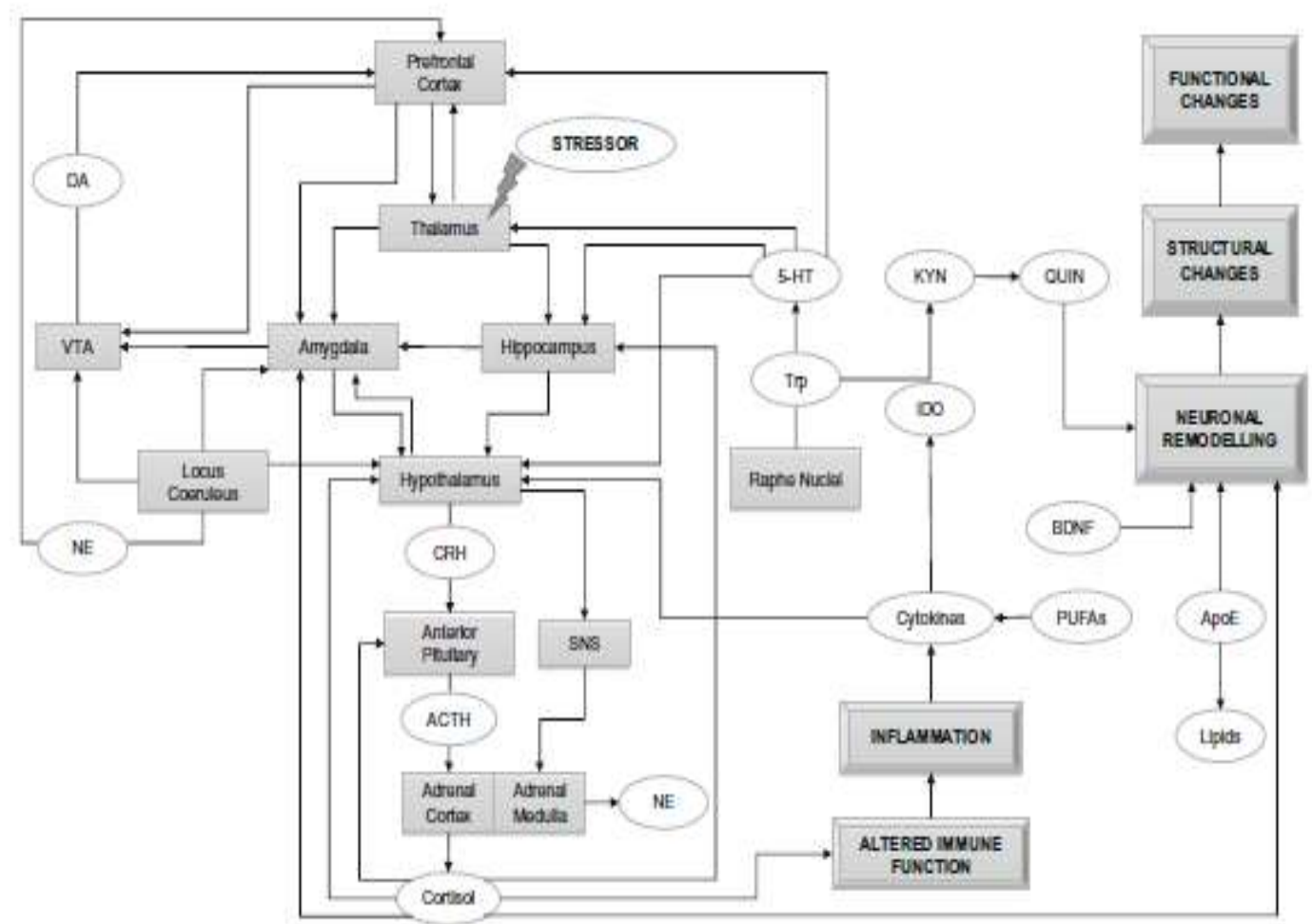

Fig.1: biomarkers associated to suicidal behavior (from Sudol et al., 2017)

It has long been known that the HPA axis hyperactivity is a risk factor for suicide attempt. Some studies reported that saliva cortisol levels were lower not only among suicide attempters relative to non attempters but also in non-attempters with previous suicide behavior. (Chatzittofis et al., 2013). It has been suggested that HPA axis anomaly may drive suicidal behavior through a concomitantly serotonin hypofunction (Coryell and Schlesser et al., 2007), especially involving 5-HT receptors and 5-HTT. In this regard many studies have hypothesized that the interaction between HPA-axis and 5-HT system may be explained by a genetic and endophenotypic vulnerability (impaired ability to respond to ongoing stressors by the offspring of relatives with mental disorders) that, combined with early childhood stressors, leads to increased risk for suicide behavior (Melhem et al., 2016; Perroud et al., 2016). The 5-HT system has also been studied extensively since the connection with some markers of inflammation. Interestingly, many studies have examined two pro-inflammatory metabolites from the serotonin precursor tryptophan, kyneurine and quinolinic acid. The assumption 
could be related to a shunt away from serotonin toward kyneurine and quinolinic acid leading to an activation of neuroinflammation by IL-6 (Isung et al.,2014). It's noteworthy higher levels of two pro-inflammatory molecules in suicide attempters than non-attempters. Moreover among the suicidal attempters, subjects with violent attempts had higher levels of kyneurine and quinolinic acid than those with non-violent ones (Bradley et al., 2015). Nevertheless another inflammatory pathway of increasing risk of suicide behavior may be related to the activation of neuro-oxitadive and neuro-nitrosative stress, through lowered levels of antioxidants and oxidant enzymes, which in turn is related to the loss of neural function by oxidation of membrane lipids (Vergas et al., 2013;). This mechanism may be particularly related to suicide attempts among mentally disordered subjects exposed to early life trauma (Moraes et al., 2018).

In summary it is likely that genetic vulnerability next to early life traumas may modulate both the serotonergic system and HPA-axis resulting in higher cortisol levels and drop-down inflammatory processes. However, to date, no single biomarker could be a strong predictive value in relation to the risk of suicide behaviors. The most promising biomarkers of suicide attempt may arise from serotoninergic system and blood measures of the inflammasome, lipids and possibly BDNF. The downstream possible linkage between lipid metabolism and suicide attempt will be detailed within the next chapter. 


\section{CHAPTER 2}

\section{LIPIDS AND SUICIDE ATTEMPT}

\subsection{LIPID LOWERING DRUGS AND RISK OF SUICIDE ATTEMPT}

The possible links between low serum lipids and suicide were initially supposed when an increased mortality from suicide, accident or trauma (non-illness mortality) was observed following the use of cholesterol-lowering drugs (Hibbeln and Salem,1996; Yang et al., 2003). In particular a metaanalysis (Muldoon et al., 1990) including 103 deaths due to non cardiovascular reasons among men from six large primary prevention trials highlighted that deaths from accidents, violence or suicide were increased by the HMG-CoA reductase inhibitors (statins) and cholesterol lowering diet $(\mathrm{OR}=1.76 ; 95 \% \mathrm{CI}, 1.19-2.58)$. However a subsequent meta-analysis including more than 70.000 subjects (Muldoon et al., 2001) showed no increase of mortality from non-illness causes by cholesterol low treatment $(\mathrm{OR}=0.84 ; 95 \% \mathrm{CI}, 0.50-1.41)$ although a modest rise was observed among subjects treated with dietary interventions and non-statin drugs (OR=1.32; 95\% CI,0.981.77) (Fig.2).

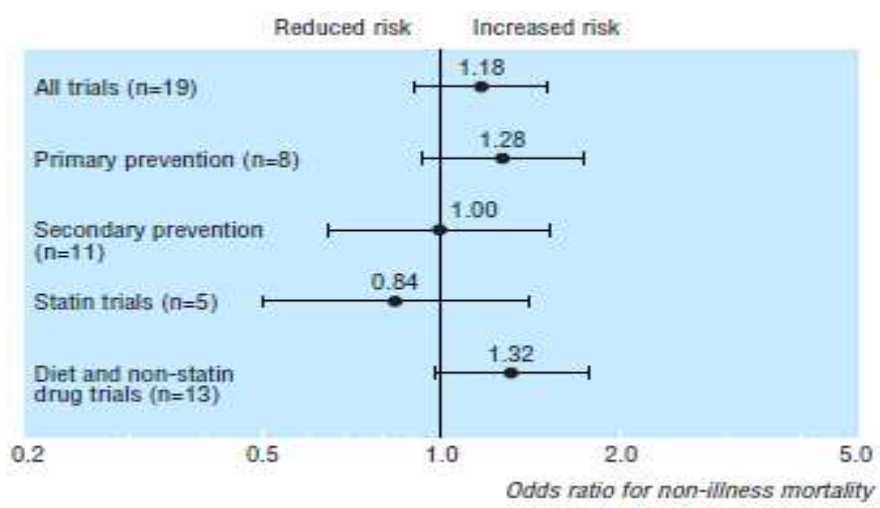

Fig.2: odds ratio and $95 \%$ confidence intervals of non-illness mortality on randomized clinical trials of cholesterol reduction (from Muldoon et al., 2001)

Furthermore, a subsequent case-control study (Yang et al.,2003) within the United Kingdom General Practice Research Database showed no association between the use of statins and other lipid-lowering drugs and depression o suicide. Another later case-control study (Leppien et al.,2018) found that total cholesterol levels but not statin therapy were related to aggressive 
behavior. Despite the conflicting results of the studies, neuropsychiatric adverse drug reaction including aggression, mood changes, violence and intentional injury, have been reported in some observational studies and pharmacovigilance databases concerning lipid-lowering drugs (Tatley et al., 2007). However potential confounders in the relationship between lipid-lowering drugs use and behavior changes were often not considered. For example lack of exercise is a risk factor for cardiovascular accidents and has been found to be associated with depression. Because hyperlipidemic subjects having this risk factor may be more likely to receive statins than hyperlipidemic subjects without, not controlling for physical exercise should result in an apparent association between statin use and depression (Concklin et al., 2007). Nevertheless it is widely debated the mechanism potentially related to non-illness mortality and lowering-lipid drugs, as some investigators reported an alteration of PUFA levels (Leppien et al., 2018), others impaired sleep, reduced testosterone and alteration on oxidative stress (Cham et al., 2016). In light of these gaps, in the last years a growing number of studies has investigated the possible relationship between lower serum lipid levels and suicidal behavior on the grounds that suicidal behaviors is more likely to be not a result of lipid lowering therapies, but simply of lower lipid levels as such (da Graca Cantarelli et al., 2014; Leppien et al., 2018).

\subsection{POSSIBLE NEUROBIOLOGICAL LINKS}

Cholesterol is an insoluble lipid that appears soluble in the form of lipoproteins in the bloodstream. VLDL,LDL,HDL are the most common lipoprotein and transfer lipids across the body. Total cholesterol reflects the sum of VLDL,LDL,HDL while triglycerides are another lipid derived from glycerol and fatty acids. VLDL,LDL and triglycerides contain pro-atherogenic lipids whilst HDL includes anti-atherogenic lipids (Baek et al.,2014) Brain cholesterol represents a quarter of the total body cholesterol and it is, apparently, produced independently from the extra-brain sources because extra-brain cholesterol does not cross the blood-brain barrier (Schreurs et al., 2010). More than 70\% of the cholesterol is produced by the oligodendrocyte to form the myelin sheat, while astrocytes and microglia contain $20 \%$ and neurons $10 \%$ of the brain cholesterol (da Graca Cantarelli et al., 2014). 
Astrocytes yield enzymes for cholesterol synthesis and express the apolipoproteins ApoE, ApoD and ApoJ (or clusterin) necessary to bring out cholesterol (or some precursors) to the neurons by ADLs (Fig.3-A). Then the neuron uptake of these lipoproteins is via LDL-R and LRP-1. Subsequently, together with sphingolipids and gangliosides, cholesterol is grouped in microdomians of the cell membrane (less than $0.1 \mu \mathrm{m}$ ) called lipid rafts. Lipid rafts concentrate receptors, enzymes and G-proteins and are involved in several processes, as the signal transduction, intracellular trafficking and communication with cytoskeleton (Fig.3-B) (da Graca Cantarelli et al., 2014).
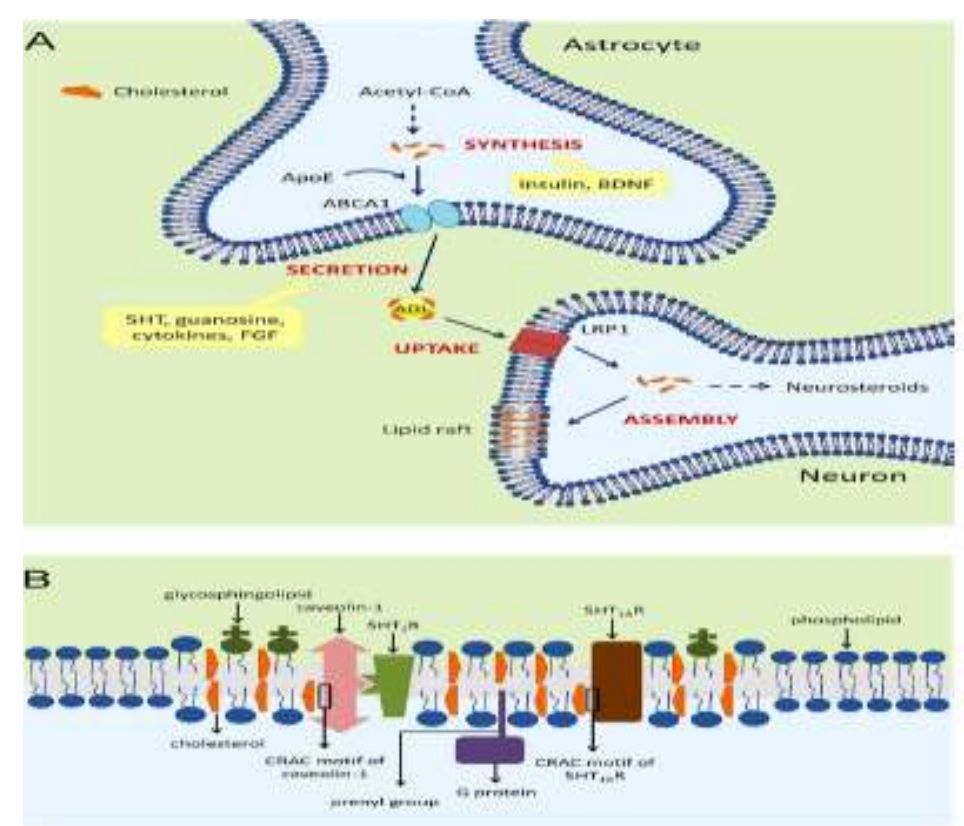

Fig.3: (A) Representation of cholesterol flux between astrocytes and neurons (B) Lipid raft containing serotonin receptors (from da Graca Cantarelli et al., 2014)

Although a relationship between low serum cholesterol levels and especially violent suicide attempt, has been observed in some observational studies (see the next subchapter), it remains to be understood how peripheral cholesterol affects brain cholesterol. It is possible that low peripheral cholesterol alteration accompanies the cholesterol changes occurring in specific synaptic rafts via metabolites of cholesterol as 27-hydroxycholesterol (Wallner et al., 2009). However this issue is very complex and the literature provides many conflicting results (Kim and Lee, 2017). Despite controversial results about the link between central and peripheral cholesterol, many studies have shown that changes of lipid raft due to alterations in lipid trafficking, lipoprotein-mediated lipid 
recycling and imbalance in the cholesterol/phospholipid in the synaptosome may alter the synaptic transmission and neural plasticity (Wang et al., 2008; Freemantle et al., 2013). In particular changes in viscosity of lipid raft by a decrease of membrane cholesterol may impair serotoninergic activity leading to an increasing risk of suicide behavior (Jafurulla et al., 2011). Serotonin is a key neurotransmitter involved in a large variety of physiological function like mood, cogntion and impulsivity. Thus, it is not surprising that the association between lower cholesterol levels and suicide attempt has been found to be stronger in subjects who had attempted suicide with a violent method (Troisi et al., 2011). In addition other mechanisms involving the lipid raft as serotonindopamine interaction, higher levels of testosterone and corticosteroids, BDNF decrease, changes of glial derived protein S100B levels and alteration in the ratio of omega-3 and omega-6 PUFAs have been proposed (Voulalas et al.,2011; Pompili et al., 2017).

Although many neurochemical associations for the cholesterol-serotonin-impulsivity hypothesis are missing, it's possible that peripheral cholesterol and other related molecules (serotonin metabolites, sterols, BDNF, S100B) may be useful markers for suicide attempt (Voulalas et al.,2011). However it is still unclear whether and how different subfractions of cholesterol (VLDL, LDL,HDL and triglycerides) have the same effect on serotonin system (Baek et al., 2014).

\subsection{LIPID ALTERATIONS IN SUICIDE ATTEMPT IN SUBJECTS WITH MENTAL DISORDERS: REVIEW OF THE LITERATURE}

The involvement of lipids in suicide is a debated issue in current research. Several studies have reported that the risk of dying from suicide attempt is higher in subjects with lower serum cholesterol levels. However the literature is controversial and, to date, there is not an agreement on possible usefulness of lipids as biological markers of suicide behaviors.

\section{Meta-analyses}

To date, three meta-analysis have explored the possible association between serum lipid levels and suicide. The first (Lester et al., 2002) reported a weak statistically significant increased risk of 
suicide in subjects with lower cholesterol levels among 10 longitudinal studies of people with known cholesterol levels (d.f. $=469.633$; chi-square $=0.005 ; \mathrm{p}<0.001)$. Moreover, examining 18 casecontrols studies, the serum cholesterol levels of suicide attempters were lower than non-attempters psychiatric controls (d.f. $=5.083$; chi-square $=0.062 ; \mathrm{p}<0.05)$ and this association was stronger in subjects using violent methods of suicide attempts (d.f. $=484$; chi-square $=0.158 ; \mathrm{p}<0.01)$ (Lester et al., 2003). A latest meta-analysis (Wu et al., 2015), based on 65 studies accounting for 510.392 subjects, reported that individuals who suicide attempters had significantly lower levels of serum triglycerides $(\mathrm{WMD}=-23.40 ; \mathrm{I}-\mathrm{squared}=75.1 \% ; \mathrm{p}<0.001)$ (Fig.4), total cholesterol $(\mathrm{WMD}=-22.35$; I-squared=88.5\%; $<<0.001)($ Fig.5), LDL (WMD $=-19.56 ;$ I-squared=78.5\%; $<<0.001)$ (Fig.6) but not HDL cholesterol (WMD=-0.16; I-squared=35.6\%; $\mathrm{p}=0.051)$ (Fig.7) as compared with nonsuicidal counterpart. In particular subgroups analysis showed an association between serum lipid levels and suicidal behaviors in major depressive disorder (triglycerides: WMD=-22.35; Isquared $=79.9 \% ; p=<0.001$; total cholesterol: $\mathrm{WMD}=-24.47 ;$ I-squared $=92.9 \% ; \mathrm{p}=<0.001 ;$ LDL: $\mathrm{WMD}=-13.35 ; \mathrm{I}-\mathrm{squared}=79.2 \% ; \mathrm{p}=<0.001$; HDL: not significant), especially among subjects younger than 40 years. Furthermore the lower total cholesterol levels category was related with a $123 \%$ (95\% CI, 24-302\%) higher risk of suicide attempt, if compared with the category of highest serum cholesterol levels. However a major limitation of the meta-analysis was the extremely high heterogeneity across studies according to different study designs and scale or methods used to define suicidality. 


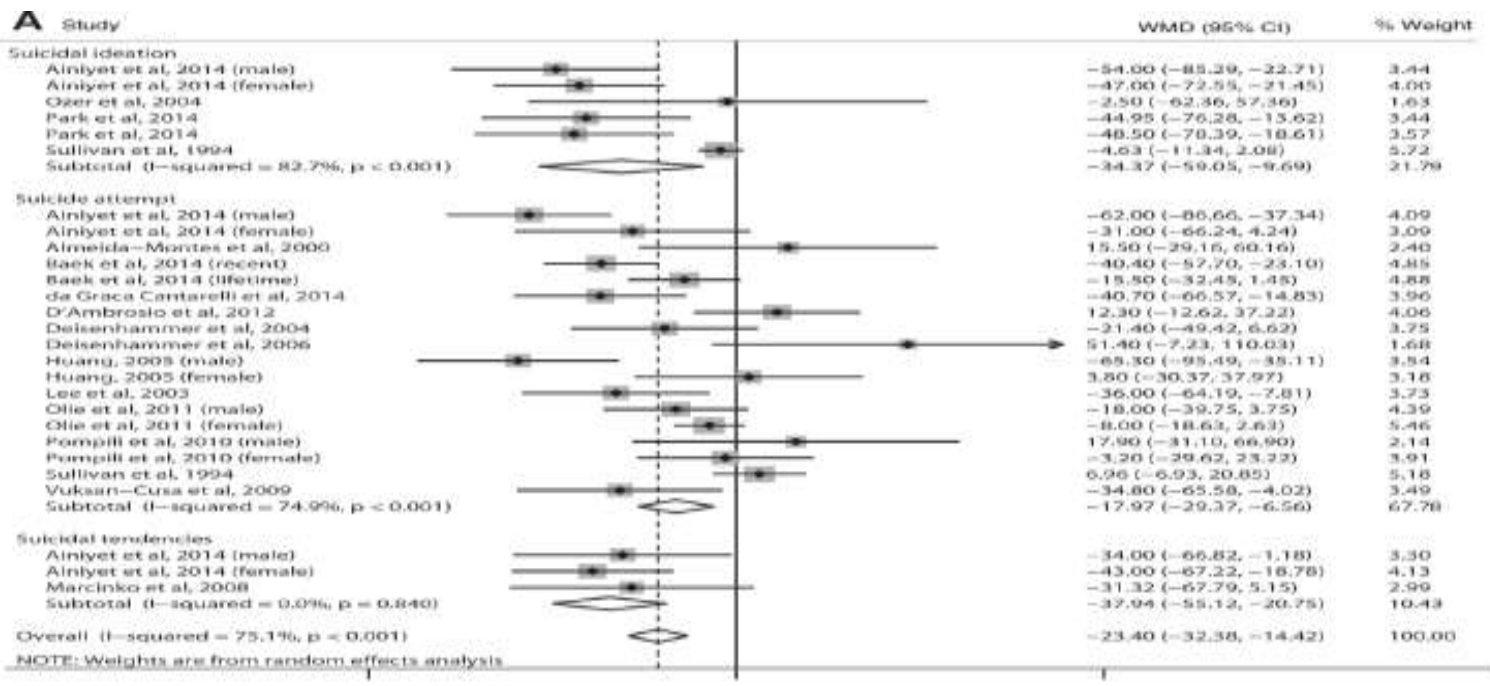

Fig 4.: Pooled summary estimates of serum triglycerides levels in suicidal versus non suicidal subjects (from Wu et al., 2015)

A

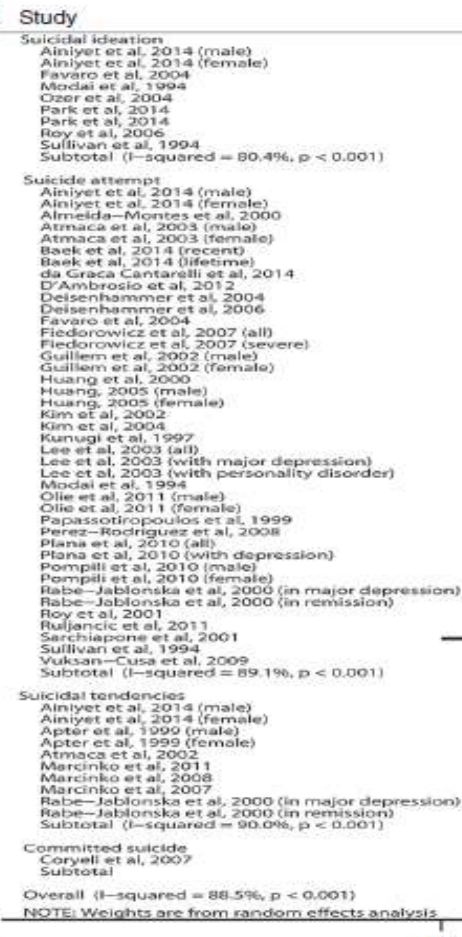

WMD ( $95 \% \mathrm{Cl})$

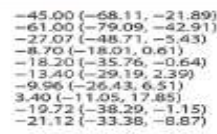

$-43.00(-64.64,251.36)$
$-5200(-68.15,-235.85)$

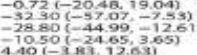

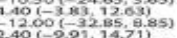

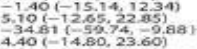

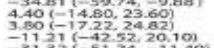
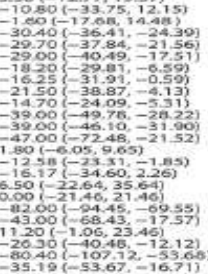

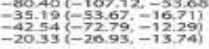
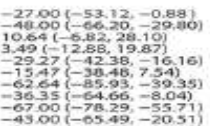

$-2830(-51.92,552)$

$22.35(-27.95,-16.75)$

1

Fig 5.: Pooled summary estimates of serum total cholesterol levels in suicidal versus non suicidal patients (from Wu et al. 2015) 
Ozeret at, 2004

Porket al, 2019

Park ot al, 2014

Roy ot al, 2006

,

Sulcide attempt

Ainiyet et al, 2014 (male)

Ainiyet et al, 2014 (female)

Almeida-Montes et at, 2000

Baek et al, 2014 (recents

Baek et al, 2014 (lifetime)
da Graca Cantarelli et al, 2014

Deisentammer et al, 2006

Huang, 2005 (male)

Hoang. 2005 tfemale

Lee ot al, 2003 (ail)

Lee et al, 2003 (with personality disonden

Rabe-Jabionska et al, 2000 in major dep

Vuksan Cusa et al, 2009

$77.9 \%, p<0.0011$

Ainiyet et al, 2014 (maie)

Ainiyet ot al, 2014 (fomet

Marcinko et al. 2008
Rabe-Jabionska ot at, 2000 (in major depression) Rabe Jablonska et al, 2000 (in remissian

Overall (1-squared $=78,5 \%, \mathrm{p}<0.001$ )

NOTE: Weights are from random effects analysis

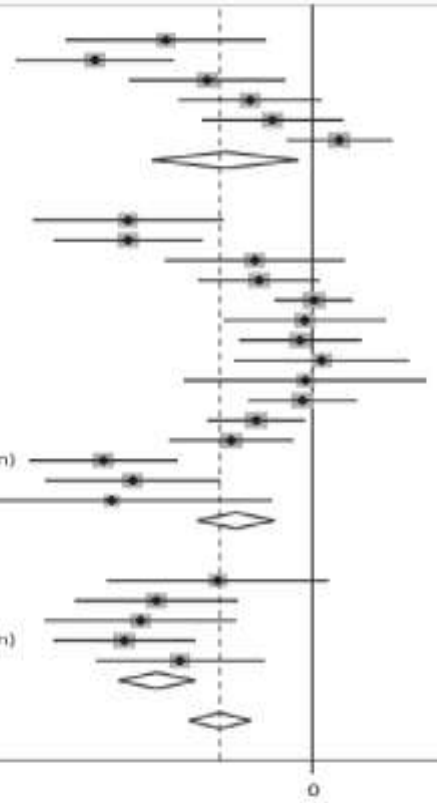

\begin{tabular}{|c|c|}
\hline$-3100(-52.26,-9.74)$ & 3.36 \\
\hline$-46.00(-62.60 ;-29.32)$ & 3.08 \\
\hline$-22.90(-38.83,-5.77)$ & 3.00 \\
\hline$-13.18(-28.34,1.98)$ & 4.06 \\
\hline$-0.46(-23.45,6.53)$ & 4.08 \\
\hline $5.70(-5.53,16.93)$ & 4.49 \\
\hline$-18.42(-33.90,-2.95)$ & 23.76 \\
\hline$-39.00(-59.15,-18.85)$ & 3.48 \\
\hline$-30.00(-54.73,-23.27)$ & 3.00 \\
\hline$-12.21(-31.30,6.88)$ & 3.60 \\
\hline$-11.30(-24.19,1.59)$ & 4.31 \\
\hline $0.30(-7.94,0.54)$ & $4.7 n$ \\
\hline$=1.65(-18.81,15.51)$ & 3.82 \\
\hline$-2.60(-15.56,10.36)$ & 4.31 \\
\hline $1,90(-16.00,20,40)$ & 3.67 \\
\hline$-1.50(-27.18,24.18)$ & 2.89 \\
\hline$-2.10(-13.61,9.41)$ & 4,46 \\
\hline$-11.00(-22.27,-1.51)$ & 4.5n \\
\hline$-17.19(-30.31,-4.07)$ & 4.29 \\
\hline$-44.20(-59.86,-28.54)$ & \\
\hline$-3800(-5655,-1045)$ & 3.66 \\
\hline$-42.47(-76.33,-8.61)$ & 2.18 \\
\hline$-16.17(-24.39,-7.94)$ & 58.04 \\
\hline$-2000(-43,46,3,46)$ & 3.12 \\
\hline$-33.00(-50.28,-15.72)$ & 3.81 \\
\hline$-36.35(-56.69,-16.01)$ & 3.46 \\
\hline-70 . & 4.08 \\
\hline$-28.00(-45.86,-10.14)$ & 3.74 \\
\hline$-32.94(-41.06,-24.82)$ & 18.20 \\
\hline$-19.56(-20.19,-12.99)$ & 100.00 \\
\hline
\end{tabular}

$-78.8$

76.9

Fig. 6: Pooled summary estimates of serum LDL levels in suicidal versus non suicidal subjects (from Wu et al., 2015)

A study

Suicidal ideation

Ainiyet et al, 2014 (male)

Ainiyet et al, 2014 (female)

Ozer et al, 2004

Parket al, 2014

Park et al, 2014

Subtotal (1-squared $=0.0 \%, p=0.959$ )

Suicide attempt

Ainiyet et al, 2014 (male)

Ainiyet et al, 2014 (female)

Almeida-Montes et al, 200

Baek et al, 2014 (recent)
Baek et al, 2014 (lifetime)

Baek et al, 2014 (lifetime)

D'Ambrosio et al, 2012

Deisenhammer et al, 2004

Deisenhammer et al, 2006

Huang, 2005 (male)

Huang, 2005 (female)

Maes et al, 1997

Vuksan-Cusa et al, 2009

Subtotal (1-squared $=40.4 \%, p=0.065$ )

Suicidal tendencies

Ainiyet et al, 2014 (maie)

Ainiyet et al, 2014 (female)

Marcinko et al, 2008

Subtotal $(1$-squared $=61.1 \%, p=0.076$

Overall (1-squared $=35.6 \%, p=0.051$ )

NOTE: Weights are from random effects analysis

$-75.5$
WMD $(95 \% \mathrm{Cl}) \quad \%$ Weight

$0.00(-6.89,6.89) \quad 5.12$

$-3.00(-9.21,3.21) \quad 5.80$

$1.70(-9.19,12.59) \quad 2.63$

$1.29(-9.58,12.16) \quad 2.64$

$0.15(-6.82,7.12) \quad 5.05$

$-0.26(-3.13,2.62) \quad 289$

$10.00(-3.09,23.09) \quad 1.93$

$-5.00(-13.71,3.71) \quad 3.72$

$1.17(-5.39,7.73) \quad 5.44$

$10.20(3.00,17.40) \quad 4.84$

$3.80(-0.83,8.43) \quad 7.82$

$-0.89(-7.58,5.80) \quad 5.31$

$1.10(-3.15,5.35) \quad 8.40$

$3.00(-3.61,9.61) \quad 5.39$

$-5.50(-16.31,5.31) \quad 2.66$

$3.70(-16.87,24.27) \quad 0.85$

$-0.20(-6.74,6.34) \quad 5.46$ $-7.90(-14.73,-1.07) \quad 5.18$

$7.73(-60.08,75.54) \quad 0.08$

$0.93(-1.80,3.65)-57.08$

$3.00(-5.38,11.38) \quad 3.93$

$-5.00(-11.19,1.19) \quad 5.82$

$-9.66(-16.73,-2.59) \quad 4.95$

$-4.24(-10.87,2.40) \quad 14.70$

$-0.16(-2.13,1.80) \quad 100.00$

Fig.7: Pooled summary estimates of serum HDL levels in suicidal versus non suicidal subjects (from Wu et al., 2015)

Finally a meta-analysis including 11 studies of 1042 individuals affected by bipolar disorder found no differences in total cholesterol ( $\mathrm{SMD}=-0.10 ; 95 \% \mathrm{CI},-0.30$ to $-0.10 ; \mathrm{p}=0.34$ ), LDL cholesterol $(\mathrm{SMD}=-0.26 ; 95 \% \mathrm{CI},-0.65$ to $0.13 ; \mathrm{p}=0.19)$ and triglycerides profile $(\mathrm{SMD}=-0.06 ; 95 \% \mathrm{CI},-0.31$ to $0.19 ; \mathrm{p}=0.63$ ) between suicide attempters and non-attempters (Bartoli et al., 2017a). Despite 
some methodological differences across the included studies, the overall estimated statistical heterogeneity was low-moderate (Fig.8).

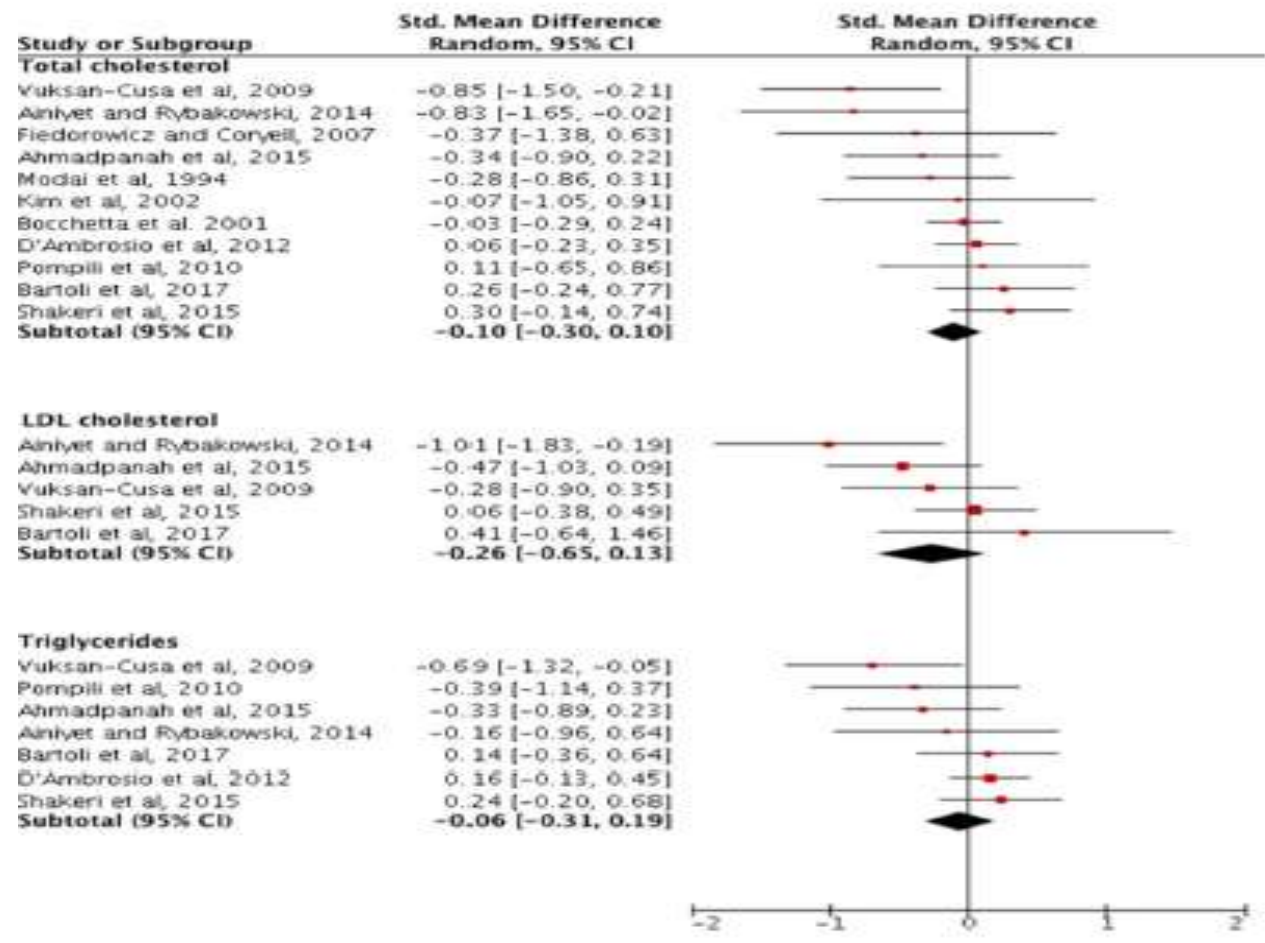

Fig.8: Forest plots: lipid profile and suicide attempt in bipolar disorder (from Bartoli et al., 2017a)

\section{Observational studies}

To date, studies exploring the association between lipid serum levels and suicide attempt in subjects with mental disorders produced mixed results (Table 1). In particular high methodologically heterogeneity across studies is obvious for many reasons. Above all the assessment of suicide attempt is different among studies, both in terms of definition (e.g. the distinction between lethality and/or intent to die) and in terms of temporality. Secondly, some studies include subjects with different mental disorders, others relate lipid serum levels to specific psychological construct as anhedonia, impulsivity, alexithymia (Loas et al., 2016). Thirdly, lipid serum levels are often investigated as a parameter discriminating between non-suicidal and suicidal subjects in terms of dichotomization rather than continuous variable. In this regard some authors (Kim and Myint, 2004) have conducted a ROC analysis finding a serum total cholesterol level of $160 \mathrm{mg} / \mathrm{dl}$ as a good cutoff point with moderate sensitivity and specificity. Moreover, as some case-control studies lack of psychiatric controls, it is difficult making a comparison of findings among the studies 
(Papadopolou et al.,2013).Most studies have investigated the relationship between lipids and suicide attempt in depressive subjects. However inconsistent results have been reported. Some studies found a statistically significant association between low levels of total cholesterol as well as of lipoprotein subfractions and suicide attempt (Kim et al., 2002; Kim and Myint, 2004; Ruljancic et al.,2011; Messaoud et al.,2017; Rui et al., 2018) whilst further studies reported mixed results (Baek et al., 2014) or no association (Almeida-Montes et al.,2000; Park et al., 2013; Bartoli et al., 2017b). Conversely, some authors revealed a positive association between serum lipid levels and suicide attempt (Brunner et al.,2006; Baek et al., 2014). Finally, cross-sectional (Ainiyet et al.,2014; Loas et al., 2016) and cohort studies (Fiedorowicz and Coryell, 2007) including depressive subjects among MDD, bipolar and schizoaffective disorder showed different results. Similarly studies including individuals with bipolar (d'Ambrosio et al.,2012; Shakeri et al.,2014; Ahmadpanah et al.,2015), schizoaffective (Marcinko et al.,2008) or mixed mood disorders (Kunugi et al.,1997; Guillem et al., 2002; da Graca Cantarelli et al.,2015) produced controversial results. In the same way studies regarding subjects with SCZ showed mixed result (Kunugi et al.,1997; Tiao et al., 2000; Atmaca et al., 2003; Park et al., 2013; Mensi et al.,2016) whilst a recent study has shown that schizophrenia patients with suicide attempt had higher serum levels of total cholesterol and triglycerides than schizophrenia patients without suicide attempt (Tatar et al, 2018). On the other hand the only study conducted on FEP subjects (Ayesa-Arriola et al., 2018) reported a lower level of LDL cholesterol in suicidal attempters than non attempters. Few studies on personality disorders (Kunugi et al.,1997; Atmaca et al.,2002; Kim et al.,2002; Papadopolou et al.,2013) have reported a relationship between lower total cholesterol serum level and suicide attempt. Finally most studies have been conducted on different diagnostic samples (Diaz-Sastre et al, 2007; Perez-Rodriguez et al.,2007; de Leon et al.,2011; Papadopolou et al.,2013; Park et al.,2013; Perera et al.,2018) producing once again mixed results.

Some studies have been performed dichotomizing total cholesterol with lower range of values less than $160 \mathrm{mg} / \mathrm{dl}$ in accordance with previous studies (Papakostas et al.,2003; Kim and Myint, 2004). In the first, a higher proportion of the suicide attempters with cholesterol levels below the cutoff 
point of $160 \mathrm{mg} / \mathrm{dl}$ has been found, although the difference remained significant in men, but not in women (Diaz Sastre et al.,2007). In the second, (Perez-Rodriguez et al.,2008) authors found an association between lower cholesterol and past suicide attempts with adjusted OR ranging from 1.8 for female to 2.0 for male sex. Finally in another study (de Leon et al., 2011) lower total cholesterol was significantly associated with a current suicide attempt only in male sex $(O R=1.4)$. However, in one cohort study (Fiedorowicz and Coryell, 2007), total cholesterol values were divided into groups with a cutoff point of $190 \mathrm{mg} / \mathrm{dl}$, but Kaplan - Meier survival analysis did not predict the possible relationship between lower cholesterol levels and the likelihood of a next suicide attempt. 
Table1: studies on serum lipid levels and suicide attempt in subjects with mental disorder

\begin{tabular}{|c|c|c|c|c|}
\hline Authors & $\begin{array}{l}\text { Study } \\
\text { Design }\end{array}$ & N of subjects & Suicidality & Lipid levels \\
\hline Kunugi,1997 & Case-control & $\begin{array}{c}173 \text { mood } \\
\text { disorders, } \mathrm{PD}, \mathrm{SCZ} \\
\text { inpatients ( } 99 \\
\text { suicide attempters; } \\
74 \text { non suicide } \\
\text { attempters); } 39 \\
\text { controls }\end{array}$ & Recent suicide attempt & $\begin{array}{l}\downarrow \text { Total cholesterol in mood } \\
\text { disorders and PD suicide } \\
\text { attempters than non } \\
\text { attempters and controls }\end{array}$ \\
\hline $\begin{array}{c}\text { Almeida- } \\
\text { Montes, } 2000\end{array}$ & Cross-sectional & $\begin{array}{l}33 \text { MDD inpatients } \\
\text { and outpatients }\end{array}$ & $\begin{array}{l}\text { Suicide attempt in the } \\
\text { previous month }\end{array}$ & no association \\
\hline Tiao, 2000 & Cross-sectional & $106 \mathrm{SCZ}$ inpatients & Recent suicide attempt & $\begin{array}{l}\text { Total cholesterol: no } \\
\text { association }\end{array}$ \\
\hline Atmaca,2002 & Case-control & $\begin{array}{l}16 \text { PD inpatients, } \\
16 \text { controls }\end{array}$ & $\begin{array}{l}\text { Current suicide } \\
\text { thoughts, history of } \\
\text { suicide attempt }\end{array}$ & $\begin{array}{l}\downarrow \text { Total cholesterol in } \\
\text { patients with current } \\
\text { suicidal thoughts and a } \\
\text { history of suicide attempt } \\
\text { than controls }\end{array}$ \\
\hline Guillem, 2002 & Cross-sectional & $\begin{array}{l}155 \text { inpatients with } \\
\text { mood disorders }\end{array}$ & Recent suicide attempt & $\begin{array}{c}\downarrow \text { Total cholesterol in } \\
\text { female suicide attempters }\end{array}$ \\
\hline Kim,2002 & Case-control & $\begin{array}{l}\text { 231 SCZ,BD, } \\
\text { MDD, PD inpatiens } \\
231 \text { controls }\end{array}$ & Recent suicide attempt & $\begin{array}{c}\downarrow \text { Total cholesterol in MDD } \\
\text { and PD suicide attempters } \\
\text { than non attempters and } \\
\text { controls }\end{array}$ \\
\hline Atmaca, 2003 & Case-control & $\begin{array}{l}32 \text { SCZ inpatients; } \\
16 \text { controls }\end{array}$ & Recent suicide attempt & $\begin{array}{l}\downarrow \text { Total cholesterol in } \\
\text { suicide attempters than non } \\
\text { attempters and controls }\end{array}$ \\
\hline Kim, 2004 & Case-control & $\begin{array}{c}149 \text { MDD } \\
\text { inpatients, } 251 \\
\text { controls }\end{array}$ & Recent suicide attempt & $\begin{array}{l}\downarrow \text { Total cholesterol in } \\
\text { suicide attempters than non } \\
\text { attempters and controls }\end{array}$ \\
\hline Brunner,2006 & Cross-sectional & $\begin{array}{l}1205 \text { MDD } \\
\text { outpatients }\end{array}$ & $\begin{array}{c}\text { Suicide ideation and } \\
\text { attempt in the last year }\end{array}$ & $\begin{array}{c}\uparrow \text { Total cholesterol, } \\
\text { triglycerides in suicide } \\
\text { attempters }\end{array}$ \\
\hline Diaz-Sastre, 2007 & Case-control & $\begin{array}{l}177 \text { inpatients with } \\
\text { mood disorders, } \\
\text { PD, SCZ, anxiety } \\
\text { disorders; } 177 \\
\text { controls }\end{array}$ & $\begin{array}{l}\text { Recent suicide } \\
\text { attempt }\end{array}$ & $\begin{array}{c}\downarrow \text { Total cholesterol in male } \\
\text { suicide attempters than } \\
\text { controls }\end{array}$ \\
\hline Fiedorowicz, 2007 & $\begin{array}{l}\text { Prospective } \\
\text { cohort }\end{array}$ & $\begin{array}{l}74 \text { MDD inpatients } \\
\text { (unipolar, bipolar, } \\
\text { schizoaffective } \\
\text { depression) }\end{array}$ & $\begin{array}{l}\text { Three years of follow } \\
\text { up; severe suicide } \\
\text { attempt defined with } \\
\text { a score }\end{array}$ & $\begin{array}{l}\text { Total cholesterol: } \text { no } \\
\text { association }\end{array}$ \\
\hline Marcinko,2008 & Case-control & $\begin{array}{l}40 \text { inpatients with } \\
\text { schizoaffective } \\
\text { disorder; } 20 \\
\text { controls }\end{array}$ & $\begin{array}{c}\text { Suicidal ideation, } \\
\text { attempt or both (scale) }\end{array}$ & $\begin{array}{l}\downarrow \text { Total, LDL and HDL } \\
\text { cholesterol in suicide } \\
\text { attempters than non } \\
\text { attempters and controls }\end{array}$ \\
\hline
\end{tabular}




\begin{tabular}{|c|c|c|c|c|}
\hline $\begin{array}{c}\text { Perez- } \\
\text { Rodriguez,2007 }\end{array}$ & Case-control & $\begin{array}{c}572 \text { inpatients } \\
\text { with mental } \\
\text { disorder (417 with } \\
\text { suicide attempt } \\
\text { history, } 155 \\
\text { without such } \\
\text { history) } 358 \\
\text { controls }\end{array}$ & $\begin{array}{c}\text { History of suicide } \\
\text { attempt }\end{array}$ & $\begin{array}{c}\downarrow \text { Total cholesterol in } \\
\text { suicide attempters than } \\
\text { non attempters and } \\
\text { controls }\end{array}$ \\
\hline de Leon, 2011 & $\begin{array}{c}\text { Cross- } \\
\text { sectional }\end{array}$ & $\begin{array}{l}1284 \mathrm{SCZ}, \mathrm{MDD} \\
\text { inpatients }\end{array}$ & $\begin{array}{l}\text { Recent and past } \\
\text { history of suicide } \\
\text { attempt }\end{array}$ & $\begin{array}{c}\downarrow \text { Total cholesterol in male } \\
\text { suicide attempters }\end{array}$ \\
\hline Ruljancic, 2011 & Cross-sectional & $132 \mathrm{MDD}$ & Recent suicide attempt & $\begin{array}{l}\downarrow \text { Total cholesterol in } \\
\text { suicide attempters }\end{array}$ \\
\hline D'Ambrosio,2012 & Cross-sectional & $220 \mathrm{BD}$ inpatients & $\begin{array}{l}\text { Life-time suicide } \\
\text { attempt }\end{array}$ & no association \\
\hline Papadopolou,2013 & Case-control & $\begin{array}{l}\text { 51 SCZ, MDD, PD } \\
\text { inpatients with } \\
\text { recent suicide } \\
\text { attempt ; } 98 \\
\text { controls }\end{array}$ & Recent suicide attempt & $\begin{array}{c}\downarrow \text { Total cholesterol in } \\
\text { suicide attempters than } \\
\text { healthy control }\end{array}$ \\
\hline Park, 2013 & Cross-sectional & $\begin{array}{c}516 \text { BD,MDD, SCZ } \\
\text { inpatients }\end{array}$ & Recent suicide attempt & $\begin{array}{l}\text { Total and HDL cholesterol, } \\
\text { triglycerides: no association }\end{array}$ \\
\hline Ainiyet,2014 & Cross-sectional & $\begin{array}{c}223 \text { BD (depression } \\
\text { phase), MDD } \\
\text { inpatients }\end{array}$ & $\begin{array}{l}\text { Suicidal thoughts, } \\
\text { tendencies and/or } \\
\text { attempts in the last } 3 \\
\text { months before } \\
\text { admission }\end{array}$ & $\begin{array}{c}\downarrow \text { Total and LDL } \\
\text { cholesterol, triglycerides } \\
\text { associated with suicidal } \\
\text { thoughts, tendencies and } \\
\text { attempts }\end{array}$ \\
\hline Baek,2014 & Cross-sectional & $\begin{array}{l}555 \mathrm{MDD} \\
\text { outpatients }\end{array}$ & $\begin{array}{l}\text { Life-time or suicide } \\
\text { attempt in the last } \\
\text { month; suicide ideation } \\
\text { in the last month }\end{array}$ & $\begin{array}{c}\downarrow \text { triglycerides and } \uparrow \mathrm{HDL}, \\
\text { VLDL in recent suicide } \\
\text { attempters than lifetime and } \\
\text { never attempters }\end{array}$ \\
\hline Shakeri,2014 & Cross-sectional & 84 BD inpatients & $\begin{array}{l}\text { Lifetime suicide } \\
\text { attempt }\end{array}$ & $\begin{array}{c}\text { 个Total cholesterol in suicide } \\
\text { attempters }\end{array}$ \\
\hline Ahmadpanah,2015 & Cross-sectional & $\begin{array}{l}50 \text { BD inpatients } \\
\text { (manic or } \\
\text { depressive phase) }\end{array}$ & Recent suicide attempt & no association \\
\hline $\begin{array}{c}\text { daGraca } \\
\text { Cantarelli,2015 }\end{array}$ & Cross-sectional & $\begin{array}{l}86 \mathrm{BD}, \mathrm{MDD} \\
\text { inpatients }\end{array}$ & $\begin{array}{l}\text { Suicide attempt in the } \\
\text { last } 15 \text { days }\end{array}$ & $\begin{array}{l}\downarrow \text { triglycerides in suicide } \\
\text { attempters; Total, HDL and } \\
\text { LDL cholesterol: no } \\
\text { association }\end{array}$ \\
\hline Loas, 2016 & Cross-sectional & $\begin{array}{l}122 \text { inpatients ( } 2 \\
\text { bipolar depression, } \\
35 \mathrm{MDD}, 85 \\
\text { anxiety disorder) }\end{array}$ & $\begin{array}{c}\text { Suicidal ideation } \\
\text { (scale) and attempt } \\
\text { (within the past } 15 \\
\text { days) }\end{array}$ & no association \\
\hline Mensi, 2016 & Case-control & $\begin{array}{c}126 \mathrm{SCZ} \\
\text { outpatients; } 131 \\
\text { controls }\end{array}$ & $\begin{array}{l}\text { Suicide attempt ( life- } \\
\text { time, recent) }\end{array}$ & $\begin{array}{c}\downarrow \text { Total cholesterol in SCZ } \\
\text { subjects after a recent } \\
\text { suicide attempt than SCZ } \\
\text { without suicide attempt and } \\
\text { with life-time suicide } \\
\text { HDL and LDL cholesterol, } \\
\text { triglycerides: no association }\end{array}$ \\
\hline Bartoli,2017b & Cross-sectional & $\begin{array}{l}214 \mathrm{MDD} \\
\text { inpatients }\end{array}$ & Recent suicide attempt & no association \\
\hline
\end{tabular}




\begin{tabular}{|c|c|c|c|c|}
\hline Messaoud,2017 & Case-control & $\begin{array}{l}162 \mathrm{MDD} \\
\text { inpatients; } 151 \\
\text { controls }\end{array}$ & Recent suicide attempt & $\begin{array}{l}\downarrow \text { Total cholesterol in } \\
\text { suicide attempters than non } \\
\text { attempters and controls }\end{array}$ \\
\hline Ayesa-Arriola, 2018 & Cross-sectional & 383 FEP inpatients & $\begin{array}{c}\text { Recent complete } \\
\text { suicide or attempt }\end{array}$ & $\begin{array}{c}\downarrow \text { LDL cholesterol in those } \\
\text { who completed or } \\
\text { attempted suicide }\end{array}$ \\
\hline Perera,2018 & Cse-control & $\begin{array}{l}188 \text { inpatients with } \\
\text { mental disorder ( } 84 \\
\text { with suicide attempt } \\
\text { history, } 104 \text { without } \\
\text { such history), } \\
\text { 93controls }\end{array}$ & $\begin{array}{l}\text { Suicide attempt in the } \\
\text { last } 3 \text { months }\end{array}$ & $\begin{array}{l}\text { Total cholesterol: no } \\
\text { association }\end{array}$ \\
\hline Rui, 2018 & Cross-sectional & $\begin{array}{l}271 \mathrm{MDD} \\
\text { inpatients }\end{array}$ & Recent suicide attempt & $\begin{array}{l}\downarrow \text { Total cholesterol in } \\
\text { suicide attempters }\end{array}$ \\
\hline Tatar, 2018 & Cross-sectional & $\begin{array}{c}228 \mathrm{SCZ} \\
\text { outpatients }\end{array}$ & $\begin{array}{l}\text { Suicide attempt in the } \\
\text { last } 3 \text { months }\end{array}$ & $\begin{array}{l}\uparrow \text { Total cholesterol and } \\
\text { triglycerides in suicide } \\
\text { attempters (male;female: } \\
\text { only triglycerides) }\end{array}$ \\
\hline
\end{tabular}

Studies with dichotomization of total cholesterol are indicated in bold

\subsection{LIPID ALTERATIONS IN VIOLENT AND NON-VIOLENT SUICIDE ATTEMPT}

Most of the literature shows a relationship between low serum cholesterol levels and violent behaviors through an alteration of serotonergic activity leading to an increased tendency for impulsivity and aggression. It is noteworthy that a correlation between infant exposure to violence and adult violent behaviors (i.e. cycle of violence) has been found in subjects with lower cholesterol levels (Asellus et al.,2014). Consistently, several authors have suggested that serum cholesterol might be more linked to the violence of suicide attempt than to suicide itself (Atmaca et al., 2008). The meta-analysis of $\mathrm{Wu}$ et al. reported lower serum total cholesterol level among subjects with violent suicide attempts than non-violent suicidal attempters and healthy controls. Moreover the possible association between cholesterol levels and violent suicide attempts has been confirmed in studies including individuals with specific diagnosis as MDD (Kim et al., 2004), SCZ (Atmaca et al., 2003; Marcinko et al., 2004) but not BD (d'Ambrosio et al.,2012). However most studies have been involved individuals who attempted suicide from mixed diagnosis, founding controversial results (Alvarez et al.,2000; Tripodianakis et al.,2002; Vevera et al.,2002; Atmaca et al., 2008; Papadopolou, et al.,2013; da Graca Cantarelli et al.,2015) (Table 2). It's notable that gender may 
play an important role since men may be more likely to use violent methods to attempt suicide

(d'Ambrosio et al., 2012).

Table2: studies on difference in lipid serum levels between violent and non-violent suicide attempt in mentally disordered people

\begin{tabular}{|c|c|c|c|c|}
\hline Authors & $\begin{array}{l}\text { Study } \\
\text { Design }\end{array}$ & N of subjects & $\begin{array}{c}\text { Suicidality } \\
\text { (measurement of } \\
\text { lipids) }\end{array}$ & Lipid levels \\
\hline Alvarez,2000 & Case-Control & $\begin{array}{l}52 \mathrm{SCZ}, \mathrm{PD}, \text { mood } \\
\text { disorders; } 32 \\
\text { controls }\end{array}$ & Recent suicide attempt & $\begin{array}{c}\downarrow \text { Total cholesterol in } \\
\text { violent than non-violent } \\
\text { suicide attempters and } \\
\text { controls }\end{array}$ \\
\hline Tripodianakis,2002 & Case-control & $\begin{array}{c}111 \mathrm{SCZ}, \mathrm{PD}, \\
\text { MDD, adjustment } \\
\text { disorder inpatients; } \\
62 \text { controls }\end{array}$ & Recent suicide attempt & $\begin{array}{c}\downarrow \text { Total cholesterol in both } \\
\text { violent and non-violent } \\
\text { suicide attempters. No } \\
\text { difference between violent } \\
\text { and non-violent suicide } \\
\text { attempters }\end{array}$ \\
\hline Vevera, 2002 & Case-control & $\begin{array}{c}70 \text { women } \\
\text { inpatients with } \\
\text { mental disorder; } 70 \\
\text { controls }\end{array}$ & Recent suicide attempt & $\begin{array}{l}\downarrow \text { Total cholesterol in } \\
\text { violent than non-violent } \\
\text { suicide attempters and } \\
\text { controls }\end{array}$ \\
\hline Atmaca, 2003 & Case-control & $\begin{array}{c}32 \text { SCZ inpatients; } \\
16 \text { controls }\end{array}$ & Recent suicide attempt & $\begin{array}{l}\downarrow \text { Total cholesterol in } \\
\text { violent than non-violent } \\
\text { suicide attempters and } \\
\text { controls }\end{array}$ \\
\hline Kim, 2004 & Case-control & $\begin{array}{c}149 \mathrm{MDD} \\
\text { inpatients, } 251 \\
\text { controls }\end{array}$ & Recent suicide attempt & $\begin{array}{c}\downarrow \text { Total cholesterol in } \\
\text { violent than non-violent } \\
\text { suicide attempters and } \\
\text { controls }\end{array}$ \\
\hline Marcinko, 2004 & Case-control & $\begin{array}{l}26 \text { SCZ inpatients; } \\
13 \text { controls }\end{array}$ & Recent suicide attempt & $\begin{array}{c}\downarrow \text { Total cholesterol in } \\
\text { violent than non-violent } \\
\text { suicide attempters and } \\
\text { controls }\end{array}$ \\
\hline Atmaca,2008 & Case-control & $\begin{array}{l}35 \mathrm{BD}, \mathrm{MDD}, \mathrm{PD}, \\
\text { SCZ inpatients; } 20 \\
\text { controls }\end{array}$ & Recent suicide attempt & $\begin{array}{c}\downarrow \text { Total cholesterol in } \\
\text { violent than non-violent } \\
\text { suicide attempters and } \\
\text { controls }\end{array}$ \\
\hline D’Ambrosio,2012 & Cross-sectional & $220 \mathrm{BD}$ inpatients & $\begin{array}{c}\text { Life-time suicide } \\
\text { attempt }\end{array}$ & $\begin{array}{l}\text { no difference of lipid serum } \\
\text { levels in violent suicide } \\
\text { attempters compared with } \\
\text { non-violent ones }\end{array}$ \\
\hline Papadopolou,2013 & Case-control & $\begin{array}{l}51 \mathrm{SCZ}, \mathrm{MDD}, \mathrm{PD} \\
\text { inpatients; } 98 \\
\text { controls }\end{array}$ & Recent suicide attempt & $\begin{array}{l}\text { no difference of lipid serum } \\
\text { levels in violent suicide } \\
\text { attempters compared with } \\
\text { non-violent ones }\end{array}$ \\
\hline $\begin{array}{c}\text { daGraca } \\
\text { Cantarelli,2015 }\end{array}$ & Cross-sectional & $\begin{array}{l}86 \mathrm{BD}, \mathrm{MDD} \\
\text { inpatients }\end{array}$ & $\begin{array}{l}\text { Suicide attempt in the } \\
\text { last } 15 \text { days }\end{array}$ & $\begin{array}{l}\text { no difference of lipid serum } \\
\text { levels in violent suicide } \\
\text { attempters compared with } \\
\text { non-violent ones }\end{array}$ \\
\hline
\end{tabular}




\subsection{RELEVANT FINDINGS AND MAJOR LIMITATIONS OF THE STUDIES}

Many case-control and cross-sectional studies have showed lower lipid serum levels in subjects following suicide attempt compared to those without an attempt. Comparisons of suicide attempters to normal healthy control have yielded similar results. However some studies have found no association while few studies have shown a higher lipid levels in suicidal attempters than non attempters. This heterogeneity seems mainly due to the inclusion of mixed sample since only few studies have been performed on individuals with specific diagnoses. Moreover suicide attempt has been examined using different scales or definitions across studies, rather than various criteria of temporality, and only some studies have distinguished between violent and non-violent suicide attempt. This might be important since evidence suggests that individuals with violent suicide behavior are more likely to have lower lipid levels (Atmaca et al., 2008). Finally several variables as genetic factors (Jollant et al.,2010), age (Volpato et al.,2001), gender (Zhang et al., 2005), ethnicity (Tatar et al,2018), economical status (Atmaca et al., 2003), physical activity (Harris et al.,2018), smoking (Rihmer et al.,2013), dietary habit (Marcinko et al.,2008), BMI (Chang et al.,2011), psychiatric diagnosis with or without some psychological constructs (Elmslie et al.,2001), comorbid eating (Mork et al.,2017) and substance use disorders (Messaoud et al., 2017), psychiatric and non-psychiatric medications (Olie et al.,2015), comorbid medical conditions (Diaz-Sastre et al.,2007) are potential confounders in the relationship between suicide attempt and serum lipid levels. However most studies did not adjust or adjusted only for most relevant factors.

In sum, despite controversial results in the relationship between lipid serum levels and suicide attempt in subjects with mental disorders, the literature shows that cholesterol abnormalities are more substantially found among male violent suicide attempters than female attempters (DiazSastre et al, 2007; de Leon et al.,2011; Tatar et al, 2018), suggesting a possible markers for a subgroups of individuals. 


\section{CHAPTER 3}

\section{AIMS}

The aim of the study is to compare inpatients affected by different mental disorders with and without a recent suicide attempt in terms of total cholesterol, LDL and triglyceride levels. Moreover, we aimed to explore possible differences in lipid profiles among individuals experiencing a violent, rather than non-violent, recent suicide attempt. 


\section{CHAPTER 4}

\section{METHODS}

This study was drawn up following the Strengthening the Reporting of Observational studies in Epidemiology (STROBE) Statement items (von Elm et al., 2008). The research project complied with the principles of the Declaration of Helsinki regarding medical research in humans, following local research ethical requirements.

\subsection{STUDY SETTING AND ELIGIBILITY CRITERIA}

We conducted a cross-sectional study, including adults consecutively admitted, between January 2012 and December 2017, to a Psychiatric Inpatient Unit (Desio Hospital, ASST Monza, Italy). Desio Hospital covers medical and surgical inpatient services, including a 20-bed ward for acute mental illness.

We included individuals suffering from ICD-10 SCZ spectrum, type I or II BD (whether in manic/hypomanic, mixed or depressive phase), MDD, and PD. Eligible subjects were aged between 18 and 65 years, medically stable, not needing treatment for any physical condition.

Subjects suffering from other mental disorders, i.e. obsessive compulsive disorder, eating disorders, intellectual disability and organic brain syndrome were excluded. Moreover we ruled out individuals with serious physical illnesses, including hepatitis B or C, and HIV infections, or treated with antidiabetic, anticoagulant, antiplatelet, urate- and lipid-lowering agents, thyroid hormone. Finally subjects lacking of relevant information were excluded.

\subsection{DATA COLLECTION AND DEFINITION OF SUICIDE ATTEMPT}

All data were anonymously and retrospectively collected, not allowing subject identification. For subjects with multiple admissions we used the most recent clinical data. Standard demographic (age, gender, ethnicity, employment) and clinical information on ICD-10 mental illnesses, recent suicide attempts, co-occurring alcohol and substance use disorders, body mass index (BMI) and 
blood tests, ongoing medical and psychopharmacological treatments, were retrieved from electronic health records.

We differentiated inpatients admitted for a recent suicide attempt (occurring within 72 hours before hospital admission) and those without such recent history. 'Suicide attempts' were only included as self-injuries of people willing to die, while suicidal ideation or plans without any action or lowlethal self-harm behaviors, were excluded (Nock et al., 2008). We used standard definitions to differentiate violent (firearm, hanging, cutting, jumping, car exhaust, other violent methods) from non-violent (drug overdose and poisoning) attempt methods (Stenbacka and Jokinen, 2015).

\subsection{MEASURAMENT OF SERUM LIPID LEVELS}

Information on lipid serum levels was retrieved from routine blood samples drawn at approximately $8.00 \mathrm{am}$, after an overnight fasting. We used serum blood tests carried out within 24 hours after the hospitalization. Serum concentrations were examined using standard methods at the Department of Clinical Pathology of Desio Hospital, ASST Monza, Italy.

\subsection{STATISTICAL METHODS}

Sample characteristics were summarized using mean values and standard deviation (SD) for continuous variables, while frequency and percentage for categorical variables. First, we carried out univariate analysis in order to detect relevant variables associated to suicide attempt and differences between violent and violent method. The normality of continuous data was verified by using Shapiro-Wilk's test. According to this assumption, Student's $t$ test was used or in alternative nonparametric Wilcoxon-Mann-Whitney test using for unequal variances the Welch's approximation for degrees of freedom. The categorical variables were analysed via the chi-square or Fisher's Exact tests. Finally in order to estimate the influence of serum lipid profile on suicide attempts, logistic regression analyses were carried out for the whole sample and for diagnostic subgroups (i.e., SCZ, 
BD, MDD and PD), including gender and age as covariates. In addition, in order to consider potential non-linear associations, we performed a post-hoc analysis considering total cholesterol levels as categorical variable. We tested whether subjects with low total cholesterol levels $(<160$ $\mathrm{mg} / \mathrm{dL}$ ) would have higher rate of recent suicide attempts, as previously described (de Leon et al., 2011). Statistical significance was set at $p<0.05$. The statistical package used for analysis was Stata 13.1 (StataCorp, College Station, TX). 


\section{CHAPTER 5}

\section{RESULTS}

We assessed for eligibility 1454 consecutively admitted inpatients. Among these, 861 subjects were excluded because they did not meet inclusion criteria: 138 because younger than 18 or older than 65 years; 382 lacking of relevant information; 68 suffered from other mental disorders; 238 individuals had physical illnesses(among these, 123 were taking antidiabetic, anticoagulant, antiplatelet and lipid-lowering agents, thyroid hormone); 35 since blood has been drawn over 72 hours (Fig.9)

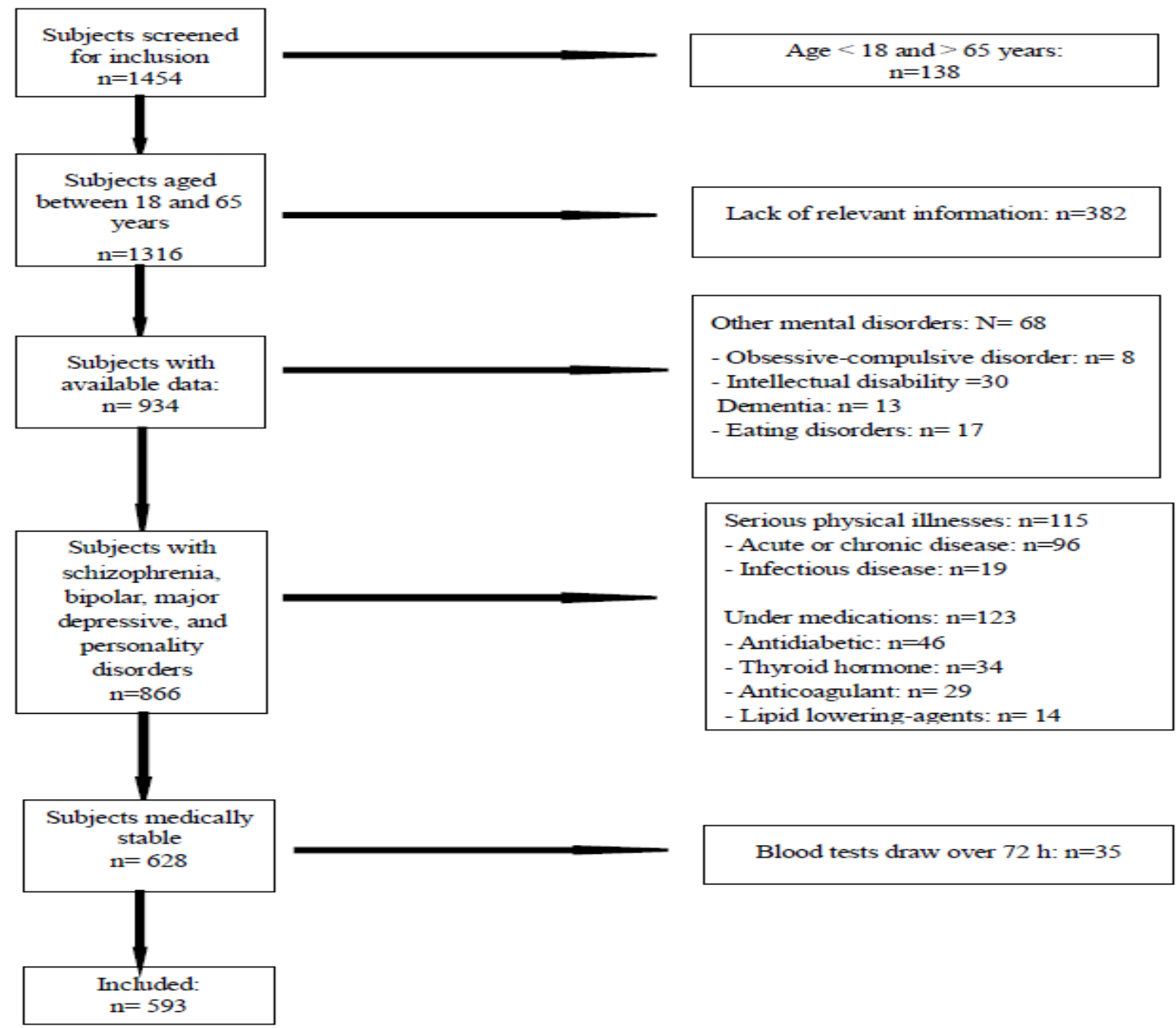

Fig. 9: Flow chart of the inclusion and exclusion of patients in the study 


\subsection{SAMPLE CHARACTERISTICS}

We included in the analysis 593 subjects (mean age \pm SD: 40.7 \pm 12.1 yrs.; female gender: 44.7\%). $12.8 \%(\mathrm{~N}=76)$ had experienced a suicide attempt in the 72 hours before admission (25 with a violent and 51 with a non-violent method). 195 subjects had SCZ, 127 BD,70 MDD and 201 PD. Alcohol (17.7\%),cannabis (10.8\%), and cocaine $(8.1 \%)$ were the most commonly misused substances. About one third of the sample was treated with second-generation antipsychotics (32.4\%). The mean BMI (SD) was $24.8(4.9) \mathrm{kg} / \mathrm{m}^{2}$. Average total cholesterol (SD) was 182.7 (41.0) $\mathrm{mg} / \mathrm{dL}$. 182 subjects were in the low cholesterol group $(<160 \mathrm{mg} / \mathrm{dL})$ while 411 in the high level group ( $\geq 160 \mathrm{mg} / \mathrm{dL})$. (Table 3). 
Table 3: Sample characteristics

\begin{tabular}{|l|c|}
\hline Variables & Sample size \\
& $\mathrm{N}=593$ \\
\hline Sociodemographic & \\
\hline Age (years) mean (SD) & $40.7(12.1)$ \\
Female gender & $265(44.7 \%)$ \\
Ethnicity (Non-Caucasian) & $50(8.4 \%)$ \\
Unemployed* & $353(59.5 \%)$ \\
\hline Recent suicide attempt & \\
\hline Non-violent & $51(8.6 \%)$ \\
\hline ICD-10 primary diagnosis & \\
\hline - SCZ & $195(32.9 \%)$ \\
- BD & $127(21.4 \%)$ \\
- MDD & $70(11.8 \%)$ \\
- PD & $201(33.9 \%)$ \\
\hline Medication before admission* & \\
\hline - FGA & $59(9.9 \%)$ \\
- SGA & $192(32.4 \%)$ \\
- LAI & $58(9.8 \%)$ \\
- Mood Stabilizers & $106(17.9 \%)$ \\
- Antidepressants & $131(22.1 \%)$ \\
\hline Smoking* & $285(48.1 \%)$ \\
\hline Comorbid substance use disorder* & \\
\hline - Alcohol & $105(17.7 \%)$ \\
- Cannabis & $64(10.8 \%)$ \\
- Cocaine & $48(8.1 \%)$ \\
- Opioids & $8(1.3 \%)$ \\
- Other & $20(3.4 \%)$ \\
\hline Metabolic characteristics mean (SD) & \\
\hline BMI (kg/m ${ }^{2} *$ & $24.8(4.9)$ \\
\hline Total cholesterol (mg/dL) & $182.7(41.0)$ \\
\hline Total cholesterol, n\% & \\
\hline$<160$ (mg/dL) & $182(30.7)$ \\
\hline$\geq 160$ (mg/dL) & $411(69.3)$ \\
\hline LDL cholesterol (mg/dL) & $110.9(36.8)$ \\
\hline Triglycerides (mg/dL) & $109.5(54.0)$ \\
\hline No. Vas a & \\
\hline
\end{tabular}

Note. Values are numbers (\%), unless stated; *There are missing values for some variables: the greatest number of missing values is for smoking and BMI, with 37.3 and 29.2 ratings, respectively. 


\subsection{UNIVARIATE ANALYSES}

No significant differences were observed in terms of sociodemographic characteristics between suicide and non-suicide attempters. There was a statistically significant difference between the two groups in diagnosis distribution and psychotropic medications with higher rate of SGA, mood stabilizers and antidepressants in the group of non-suicide attempters. Total serum cholesterol, triglycerides and LDL cholesterol levels did not differ among the two groups. However the proportion of subjects with cholesterol levels below $160 \mathrm{mg} / \mathrm{dl}$ was significantly higher in nonsuicide attempters. Full details are reported in Table 4.

Table 4: factors associated with suicide attempts: univariate analyses

\begin{tabular}{|c|c|c|c|}
\hline Variables & $\begin{array}{c}\text { Suicide } \\
\text { attempters } \\
\mathrm{N}=76(12.8 \%)\end{array}$ & $\begin{array}{c}\text { Non-suicide } \\
\text { attempters } \\
\mathrm{N}=517 \\
(87.2 \%)\end{array}$ & p-value \\
\hline \multicolumn{4}{|l|}{ Sociodemographic } \\
\hline Age (years) mean (SD) & $42.7(11.1)$ & $40.4(12.2)$ & $0.125^{\mathrm{a}}$ \\
\hline Female gender & $37(48.7 \%)$ & $228(44.1 \%)$ & $0.453^{b}$ \\
\hline Ethnicity (Non-Caucasian) & $6(7.9 \%)$ & $44(8.5 \%)$ & $0.857^{\mathrm{b}}$ \\
\hline Unemployed & $34(51.5 \%)$ & $319(66.9 \%)$ & $0.014^{b}$ \\
\hline \multicolumn{4}{|l|}{ ICD-10 primary diagnosis } \\
\hline - SCZ & $9(11.8 \%)$ & $186(36.0 \%)$ & $<0.001^{b}$ \\
\hline$-\mathrm{BD}$ & $11(14.5 \%)$ & $116(22.4 \%)$ & \\
\hline - MDD & $20(26.3 \%)$ & $50(9.7 \%)$ & \\
\hline - PD & $36(47.4 \%)$ & $165(31.9 \%)$ & \\
\hline \multicolumn{4}{|c|}{ Medication before admission } \\
\hline - FGA & $4(5.3 \%)$ & $55(11.2 \%)$ & $0.116^{b}$ \\
\hline - SGA & $12(15.8 \%)$ & $180(36.6 \%)$ & $0.000^{b}$ \\
\hline - LAI & $5(6.6 \%)$ & $53(10.8 \%)$ & $0.261^{\mathrm{b}}$ \\
\hline - Mood Stabilizers & $7(9.2 \%)$ & $99(20.1 \%)$ & $0.023^{b}$ \\
\hline - Antidepressants & $32(42.1 \%)$ & $99(20.1 \%)$ & $<0.001^{\text {b }}$ \\
\hline Smoking & $34(75.6 \%)$ & $251(76.8 \%)$ & $0.858^{\mathrm{b}}$ \\
\hline \multicolumn{4}{|c|}{ Comorbid substance use disorders } \\
\hline - Alcohol & $15(20.3 \%)$ & $90(19.1 \%)$ & $0.807^{b}$ \\
\hline - Cannabis & $7(10.0 \%)$ & $57(12.1 \%)$ & $0.615^{\mathrm{b}}$ \\
\hline - Cocaine & $5(7.1 \%)$ & $43(9.1 \%)$ & $0.589^{b}$ \\
\hline - Opioids & $0(0.0 \%)$ & $8(1.7 \%)$ & $0.605^{\mathrm{c}}$ \\
\hline - Other & $4(5.7 \%)$ & $16(3.4 \%)$ & $0.311^{\mathrm{c}}$ \\
\hline \multicolumn{4}{|c|}{$\begin{array}{l}\text { Metabolic characteristics mean } \\
(S D)\end{array}$} \\
\hline BMI $\left(\mathrm{kg} / \mathrm{m}^{2}\right)$ & $23.6(3.7)$ & $25.0(5.1)$ & $0.07^{\mathrm{a}}$ \\
\hline Total cholesterol $(\mathrm{mg} / \mathrm{dL})$ & $187.5(39.8)$ & $182.0(41.2)$ & $0.286^{\mathrm{a}}$ \\
\hline$<160(\mathrm{mg} / \mathrm{dL})$ & $15(19.7 \%)$ & $167(32.3 \%)$ & $0.027^{\mathrm{b}}$ \\
\hline LDL cholesterol (mg/dL) & $117.4(36.3)$ & $106.9(36.9)$ & $0.073^{\mathrm{a}}$ \\
\hline Triglycerides (mg/dL) & $104.8(41.6)$ & $110.2(55.6)$ & $0.951^{\mathrm{a}}$ \\
\hline
\end{tabular}

Values are numbers (\%), unless stated;

a. Wilcoxon Mann-Whitney test; b. Pearson's $\chi^{2}$ test; c. Fisher's exact test. 
Table 5 shows the comparison of selected variables among violent and non-violent suicide attempters. The statistically significant differences among two groups were observed in female gender and antidepressant use while no relationship with lipid metabolic characteristics was found.

Table 5: differences between violent and non-violent suicide attempters: univariate analyses

\begin{tabular}{|l|c|c|c|}
\hline Variables & $\begin{array}{c}\text { Violent } \\
\text { N=25(32.9\%) }\end{array}$ & $\begin{array}{c}\text { Non-violent } \\
\text { N=51 }(67.1 \%)\end{array}$ & p-value \\
\hline Sociodemographic & & & \\
\hline Age (years) mean (SD) & $43.6(12.1)$ & $42.3(10.6)$ & $0.576^{\mathrm{a}}$ \\
Female gender & $7(28.0 \%)$ & $30(58.8 \%)$ & $\mathbf{0 . 0 1 5}^{\mathrm{c}}$ \\
Ethnicity (Non-Caucasian) & $3(12.00 \%)$ & $3(5.9 \%)$ & $0.388^{\mathrm{c}}$ \\
Unemployed & $11(50.0 \%)$ & $23(52.3 \%)$ & $0.862^{\mathrm{b}}$ \\
\hline ICD-10 primary diagnosis & & & \\
\hline - Schizophrenia & $5(20.0 \%)$ & $186(7.8 \%)$ & $0.260^{\mathrm{c}}$ \\
- Bipolar disorder & $6(24.0 \%)$ & $14(27.4 \%)$ & \\
- Major depressive disorder & $9(36.0 \%)$ & $27(52.9 \%)$ & \\
- Personality disorder & $5(20.0 \%)$ & $6(11.8 \%)$ & \\
\hline Medication before admission & & & \\
\hline - FGA & $2(8.0 \%)$ & $2(3.9 \%)$ & $0.594^{\mathrm{c}}$ \\
- SGA & $4(16.0 \%)$ & $8(15.7 \%)$ & $1.000^{\mathrm{c}}$ \\
- LAI & $2(8.0 \%)$ & $3(5.9 \%)$ & $1.000^{\mathrm{c}}$ \\
- Mood Stabilizers & $3(12.0 \%)$ & $4(7.8 \%)$ & $0.678^{\mathrm{c}}$ \\
- Antidepressants & $6(24.0 \%)$ & $26(51.0 \%)$ & $\mathbf{0 . 0 2 9 ^ { \mathbf { c } }}$ \\
\hline Smoking & $14(93.3 \%)$ & $20(66.7 \%)$ & $0.070^{\mathrm{c}}$ \\
\hline Comorbid substance use disorder & & & \\
\hline - Alcohol & $2(8.3 \%)$ & $13(26.0 \%)$ & $0.122^{\mathrm{c}}$ \\
- Cannabis & $5(21.7 \%)$ & $2(4.3 \%)$ & $0.035^{\mathrm{c}}$ \\
- Cocaine & $0(0.0 \%)$ & $5(10.6 \%)$ & $0.164^{\mathrm{c}}$ \\
- Opioids & $0(0.0 \%)$ & $0(0.0 \%)$ & - \\
- Other & $0(0.0 \%)$ & $4(8.5 \%)$ & $0.295^{\mathrm{c}}$ \\
\hline Metabolic characteristics mean & & & \\
(SD) & $178.8(31.5)$ & $191.7(43.0)$ & $0.200^{\mathrm{a}}$ \\
\hline BMI (kg/m $\left.{ }^{2}\right)$ & $100.8(48.8)$ & $106.7(37.9)$ & $0.215^{\mathrm{a}}$ \\
\hline Total cholesterol (mg/dL) & & $10(19.6 \%)$ & $1.000^{\mathrm{c}}$ \\
\hline$<160$ (mg/dL) & & & \\
\hline LDL cholesterol (mg/dL) & & & \\
\hline Triglycerides (mg/dL) & & & \\
\hline
\end{tabular}

Values are numbers (\%), unless stated;

a. Wilcoxon Mann-Whitney test; b. Pearson's $\chi^{2}$ test; c. Fisher's exact test.

Sample characteristics by cholesterol levels groups are summarized in Table 6. No significant difference was observed in recent suicide attempt $(\mathrm{p}=0.085)$. 
Table 6: factors associated with total cholesterol levels groups: univariate analyses

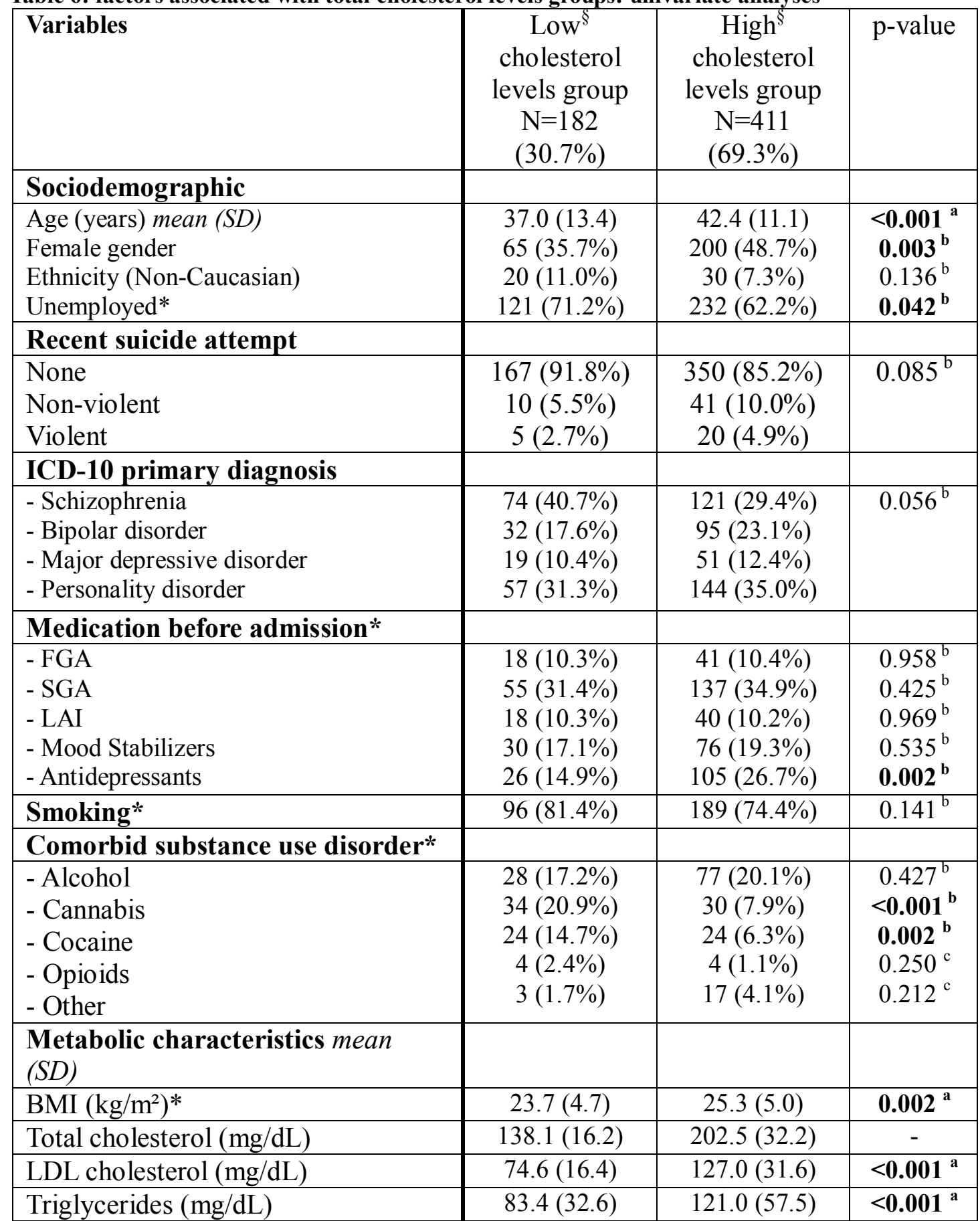

Notes. Values are numbers (\%), unless stated; ${ }^{\S}$ low cholesterol group: $<160$ (mg/dL); high cholesterol group: $\geq 160$ (mg/dL). a. Wilcoxon Mann-Whitney test; b. Pearson's $\chi^{2}$ test; c. Fisher's exact test. 


\subsection{LOGISTIC REGRESSION MODELS}

Logistic regression models, including age and gender, are shown in Table 7. Considering the whole sample, we did not find any association between recent suicide attempt and serum total cholesterol $(\mathrm{p}=0.55)$, LDL cholesterol $(\mathrm{p}=0.19)$ or triglycerides $(\mathrm{p}=0.31)$ levels, respectively. Similar findings were found considering different diagnostic subgroups.

Table 7: association between lipid profile and suicide attempt: multiple logistic regression analyses

\begin{tabular}{|l|c|c|c|c|c|c|c|c|c|c|}
\hline & \multicolumn{2}{|c|}{$\begin{array}{c}\text { Total sample } \\
\mathrm{N}=593^{\mathrm{a}}\end{array}$} & \multicolumn{2}{c|}{$\begin{array}{c}\text { Schizophrenia } \\
\mathrm{N}=195^{\mathrm{b}}\end{array}$} & \multicolumn{2}{c|}{$\begin{array}{c}\text { Bipolar disorder } \\
\mathrm{N}=127^{\mathrm{c}}\end{array}$} & \multicolumn{2}{|c|}{$\begin{array}{c}\text { Major depressive } \\
\text { disorder } \\
\mathrm{N}=70^{\mathrm{d}}\end{array}$} & \multicolumn{2}{|c|}{$\begin{array}{c}\text { Personality } \\
\text { disorder } \\
\mathrm{N}=201^{\mathrm{e}}\end{array}$} \\
\cline { 2 - 13 } & OR & $\mathrm{P}$ & $\mathrm{OR}$ & $\mathrm{P}$ & $\mathrm{OR}$ & $\mathrm{P}$ & $\mathrm{OR}$ & $\mathrm{P}$ & $\mathrm{OR}$ & $\mathrm{P}$ \\
\hline $\begin{array}{l}\text { Total } \\
\text { cholesterol }\end{array}$ & 1.002 & 0.548 & 1.002 & 0.841 & 1.000 & 0.970 & 1.000 & 0.921 & 0.999 & 0.877 \\
\hline $\begin{array}{l}\text { LDL } \\
\text { cholesterol }\end{array}$ & 1.004 & 0.189 & 1.004 & 0.682 & 1.005 & 0.538 & 1.005 & 0.519 & 1.002 & 0.621 \\
\hline Triglycerides & 0.997 & 0.310 & 0.994 & 0.515 & 0.997 & 0.630 & 0.999 & 0.896 & 0.995 & 0.225 \\
\hline
\end{tabular}

Stratified analysis across diagnostic groups. Each row represents a separate model adjusted for gender and age.

Number of suicide attempters, non-attempters: ${ }^{\mathrm{a}} 76,517 ;{ }^{\mathrm{b}} 9,186 ;{ }^{\mathrm{c}} 11,116 ;{ }^{\mathrm{d}} 20,50 ;{ }^{\mathrm{e}} 36,165$.

Finally, comparing violent and non-violent suicide attempts, no differences in total $(\mathrm{p}=0.31)$ cholesterol, LDL cholesterol $(\mathrm{p}=0.48)$, and triglycerides $(\mathrm{p}=0.27)$ serum levels could be estimated (Table 8).

Table8: association between lipid profile and violent suicide attempt: multiple logistic regression analyses

\begin{tabular}{|c|c|c|c|c|c|c|c|c|c|c|}
\hline & \multicolumn{2}{|c|}{$\begin{array}{l}\text { Whole sample } \\
\qquad \mathrm{N}=593^{\mathrm{a}}\end{array}$} & \multicolumn{2}{|c|}{$\begin{array}{l}\text { Schizophrenia } \\
\qquad N=195^{b}\end{array}$} & \multicolumn{2}{|c|}{$\begin{array}{l}\text { Bipolar disorder } \\
\qquad N=127^{c}\end{array}$} & \multicolumn{2}{|c|}{$\begin{array}{c}\text { Major depressive } \\
\text { disorder } \\
\mathrm{N}=70^{\mathrm{d}}\end{array}$} & \multicolumn{2}{|c|}{$\begin{array}{c}\text { Personality } \\
\text { disorders } \\
\mathrm{N}=201^{\mathrm{e}} \\
\end{array}$} \\
\hline & OR & $\mathbf{P}$ & OR & $\mathbf{P}$ & OR & $\mathbf{P}$ & OR & $\mathbf{P}$ & OR & $\mathbf{P}$ \\
\hline $\begin{array}{l}\text { Total } \\
\text { cholesterol }\end{array}$ & 0.992 & 0.314 & 0.980 & 0.408 & 1.019 & 0.639 & 0.992 & 0.950 & 0.984 & 0.304 \\
\hline $\begin{array}{l}\text { LDL } \\
\text { cholesterol }\end{array}$ & 0.994 & 0.478 & 0.972 & 0.308 & 1.016 & 0.672 & 1.000 & 0.992 & 0.980 & 0.238 \\
\hline Triglycerides & 0.993 & 0.268 & 0.994 & 0.785 & 0.973 & 0.372 & 1.017 & 0.177 & 0.958 & 0.076 \\
\hline
\end{tabular}

Stratified analysis across diagnostic groups. Each row represents a separate model adjusted for gender and age.

Number of violent suicide attempters, non-violent: ${ }^{\mathrm{a}} 25,51 ;{ }^{\mathrm{b}} 5,4 ;{ }^{\mathrm{c}} 5,6 ;{ }^{\mathrm{d}} 6,14 ;{ }^{\mathrm{e}} 9,27$

In addition, our post-hoc analysis showed a trend toward significance $(p=0.06)$ in the association between high cholesterol level and recent suicide attempt in the whole sample $(\mathrm{OR}=1.8)$ but not in the diagnostic subgroups (Table 9).

Furthermore, there was no statistically significant relation between method of suicide attempt and high cholesterol level both in the whole sample $(p=0.91)$ and diagnostic subgroups (Table 10). 
Table 9: association between high cholesterol level and suicide attempt: multiple logistic regression analyses

\begin{tabular}{|l|c|c|c|c|c|c|c|c|c|c|}
\hline & \multicolumn{2}{|c|}{$\begin{array}{c}\text { Total sample } \\
\mathrm{N}=593^{\mathrm{a}}\end{array}$} & \multicolumn{2}{c|}{$\begin{array}{c}\text { Schizophrenia } \\
\mathrm{N}=195^{\mathrm{b}}\end{array}$} & \multicolumn{2}{c|}{$\begin{array}{c}\text { Bipolar disorder } \\
\mathrm{N}=127^{\mathrm{c}}\end{array}$} & \multicolumn{2}{c|}{$\begin{array}{c}\text { Major depressive } \\
\text { disorder } \\
\mathrm{N}=70^{\mathrm{d}}\end{array}$} & \multicolumn{2}{|c|}{$\begin{array}{c}\text { Personality } \\
\text { disorder } \\
\mathrm{N}=201^{\mathrm{e}}\end{array}$} \\
\cline { 2 - 11 } & $\mathrm{OR}$ & $\mathrm{P}$ & $\mathrm{OR}$ & $\mathrm{P}$ & $\mathrm{OR}$ & $\mathrm{P}$ & $\mathrm{OR}$ & $\mathrm{P}$ & $\mathrm{OR}$ & $\mathrm{P}$ \\
\hline $\begin{array}{l}\text { High vs low } \\
\text { cholesterol } \\
\text { level }\end{array}$ & 1.8 & 0.057 & 1.28 & 0.740 & 4.248 & 0.168 & 0.847 & 0.780 & 2.538 & 0.076 \\
\hline
\end{tabular}

Stratified analysis across diagnostic groups. Each row represents a separate model adjusted for gender and age.

${ }^{\S}$ high cholesterol level: $\geq 160(\mathrm{mg} / \mathrm{dL})$; low cholesterol level: $<160(\mathrm{mg} / \mathrm{dL})$

Number of suicide attempters, non-attempters: ${ }^{\mathrm{a}} 76,517 ;{ }^{\mathrm{b}} 9,186 ;{ }^{\mathrm{c}} 11,116 ;{ }^{\mathrm{d}} 20,50 ;{ }^{\mathrm{e}} 36,165$.

Table 10. association between high cholesterol level and violent suicide attempt: multiple logistic regression analyses

\begin{tabular}{|l|c|c|c|c|c|c|c|c|c|c|}
\hline & \multicolumn{2}{|c|}{$\begin{array}{c}\text { Total sample } \\
\mathrm{N}=593^{\mathrm{a}}\end{array}$} & \multicolumn{2}{c|}{$\begin{array}{c}\text { Schizophrenia } \\
\mathrm{N}=195^{\mathrm{b}}\end{array}$} & \multicolumn{2}{c|}{$\begin{array}{c}\text { Bipolar disorder } \\
\mathrm{N}=127^{\mathrm{c}}\end{array}$} & \multicolumn{2}{c|}{$\begin{array}{c}\text { Major depressive } \\
\text { disorder } \\
\mathrm{N}=70^{\mathrm{d}}\end{array}$} & \multicolumn{2}{c|}{$\begin{array}{c}\text { Personality } \\
\text { disorder } \\
\mathrm{N}=201^{\mathrm{e}}\end{array}$} \\
\cline { 2 - 11 } & $\mathrm{OR}$ & $\mathrm{P}$ & $\mathrm{OR}$ & $\mathrm{P}$ & $\mathrm{OR}$ & $\mathrm{P}$ & $\mathrm{OR}$ & $\mathrm{P}$ & $\mathrm{OR}$ & $\mathrm{P}$ \\
\hline $\begin{array}{l}\text { High vs low } \\
\text { cholesterol } \\
\text { level }^{\S}\end{array}$ & 1.074 & 0.912 & 0.156 & 0.445 & 0.591 & 0.395 & 4.184 & 0.318 & 0.935 & 0.953 \\
\hline
\end{tabular}

Stratified analysis across diagnostic groups. Each row represents a separate model adjusted for gender and age.

${ }^{\S}$ high cholesterol level: $\geq 160(\mathrm{mg} / \mathrm{dL})$; low cholesterol level: $<160(\mathrm{mg} / \mathrm{dL})$

Number of violent suicide attempters, non-violent: ${ }^{\mathrm{a}} 25,51 ;{ }^{\mathrm{b}} 5,4 ;{ }^{\mathrm{c}} 5,6 ;{ }^{\mathrm{d}} 6,14 ;{ }^{\mathrm{e}} 9,27$ 


\section{CHAPTER 6}

\section{DISCUSSION}

In contrast with some hypothesizes reported in many studies, we did not find any association between serum total, LDL cholesterol, triglycerides and suicide attempt in a sample of inpatients with different mental disorder, also after considering single diagnostic subgroups. However, at the univariate analysis, the proportion of individuals with cholesterol levels above the proposed cut-off point $(160 \mathrm{mg} / \mathrm{dl})$ was statistically higher in suicide attempters than their non attempting counterparts. Furthermore, in post-hoc analyses, we apparently found a trend toward significance in the association between high cholesterol level and recent suicide attempt within the mixed sample, consistently with a recent study (Perera et al.,2018) but in opposition with others (de Leon et al., 2011). Nevertheless, this relationship may be explained by antidepressant drugs acting as potential confounders since they are associated with both suicide attempt and high cholesterol levels.

Similarly, we did not find significant differences in serum lipid levels between violent and nonviolent method of suicide attempt. However, although at a univariate level, the rate of female gender and antidepressant users were higher among non violent suicide attempters. Our result is consistent with previous studies reporting that women are far more likely than men to attempt suicide with non-violent methods and men more probable to use violent methods to attempt suicide (d'Ambrosio et al.,2012). Gender differences in the method of suicide attempt are well known and may be explained by a combination of factors including age, socio-cultural and psychological aspects, impulsivity, different rate of comorbid somatic illnesses, substance use and psychiatric disorders (Mergl et al.,2015). On the other hand, the increasing levels of brain serotonin and later anti-impulsive effect may explain the higher proportion of antidepressant medication among non violent than violent suicide attempters (Makris et al.,2013).

Several reasons might explain the discrepancies between our findings and those reported in previous studies (e.g., Ayesa-Arriola et al., 2018; Baek et al., 2014). First, we used recommended 
definitions to assess suicidal attempts and related methods, whereas previous studies were significantly heterogeneous in terms of outcome definition (suicidal thoughts, suicidal intent, suicidal plan, distinction between lethality and/or intent to die), including the related (recent or lifetime) temporality of attempt (Park et al., 2013; Wu et al., 2016). Second, we purposively considered only certain mental disorders, excluding those conditions (i.e., eating disorders, organic mental disorders and mental retardation) that might confound the association between lipid profile and suicidal behaviors (Diaz-Sastre et al., 2007). We thus carried out stratified analyses across certain psychiatric disorders, considering specific diagnostic subgroups in terms of different suicidal behavior risk (WHO, 2014). Third, some studies explored the role of specific psychological constructs, including impulsivity, alexithymia and anhedonia, when estimating the association between lipid serum levels and suicidal behaviors (de Berardis, 2008; Loas et al., 2016). Thus, it should be acknowledged that we did not consider several clinical features and complex psychological / psychosocial models, that may influence the relationship between serum lipid levels and suicide attempt (Joiner et al., 2016; Ahmadpanah et al., 2018). Finally, the impact of other biological factors influencing lipid serum levels (Bartoli et al., 2013; Correll et al., 2015) was not considered in most of previous studies, whilst we choose to rule out subjects with physical conditions, infectious disease or medical treatments, in particular lipid lowering agents, that might interfere with lipid metabolism. Therefore, our study does not support previous evidence on the association between lower serum lipid levels and suicide behaviors, regardless of specific mental disorders.

\subsection{LIMITATIONS}

This study has some limitations and results should be interpreted with prudence. First, we should consider that most subjects were treated with psychopharmacological agents, leading to metabolic side effects. In particular antidepressants could have a stronger impact on the association between serum lipid profile and suicidal behaviors than other treatments (Chocca et al.,2006; Correll et al., 2015), Nevertheless, we cannot rule out that the lack of association between serum lipid profile and 
suicide attempts might be partly explained by the fairly high BMI we estimated in our sample, possibly due to the proportion of subjects taking second-generation antipsychotics or to other factors we could not consider, such as dietary habits, nutritional status and physical activity. Second, the number of suicide attempters was relatively small, likewise the sample sizes of diagnostic subgroups, which could limit the generalizability of our results, especially considering subjects with different methods of suicide attempt and different levels of total cholesterol $(<160 \mathrm{mg} / \mathrm{dL})$. Third, since data were retrieved from electronic medical charts, without information from face to face interviews, some risk factors like previous suicide attempts, insufficient adherence to medications and specific psychopathological characteristics have not been assessed (Emet et al.,2015; Park et al.,2017). Finally, the cross-sectional design did not obviously allow any causal inference. For example, since most suicide attempts are concomitant with a depressive episode, it cannot be assessed whether a decrease in serum lipid levels may be associated with a depressive episode with subsequent suicide attempt or if this has led to loss of appetite and weight, therefore decreasing lipid levels (d'Ambrosio et al.,2012; da Graca Cantarelli et al.,2015). Thus, the poor amount of longitudinal data needs to be expanded in order to evaluate the relationship between serum lipids and suicide attempts (Fiedorowicz and Coryell, 2007). 


\section{CHAPTER 7}

\section{CONCLUSIONS AND CLINICAL IMPLICATIONS}

Despite some limitations, the current study does not support previous evidence on the relationship between individual lipid profiles and suicidal behaviors in mental disorders. Our findings did not show any statistically significant difference in cholesterol and triglycerides levels in suicide attempters as compared with non-attempters, even differentiating violent from non-violent methods. So it does not seem justifiable routine screening for cholesterol and triglycerides serum levels, in order to assess suicidal risk. However, the relationship between lipid serum levels and suicide attempt is far from clear. Further studies are needed to clarify the role of lipids as trait or state marker that may precipitate suicide attempt. Although lipid profile may represent only a part of the complex suicide risk model involving both clinical and biological factors, studies on markers of central serotonergic activity might be useful to provide a link between serum lipid levels and serotonergic system (Moraes et al.,2018). 


\section{REFERENCES}

- Ahmadpanah, M., Haghighi, M., Jahangard, L., Borzoei, S., Heshmati, S., Bajoghli, H., Holsboer-Trachsler, E., Brand, S., 2015. No evidence for metabolic syndrome and lipid profile differences in patients suffering from bipolar I disorder with and without suicide attempts. Int J Psychiatry Clin Pract 19(3), 168-173.

- Ainiyet B, Rybakowski JK.,2014. Suicidal behaviour and lipid levels in unipolar and bipolar depression. Acta Neuropsychiatr. 26(5):315-20.

- Almeida-Montes L.G.,Valles-Sanchez V., Moreno-Aguilar J., Chavez-Balderas RA., GarcíaMarín JA., Cortés Sotres JF., Hheinze-Martin G.Alvarez., 2000. Relation of serum cholesterol, lipid, serotonin and tryptophan levels to severityof depression and to suicide attempts. J Psychiatry Neurosci. 25(4):371-7

- Asellus P., Nordström P., Nordström AL., Jokinen J.,2014. Cholesterol and the "Cycle of Violence" in attempted suicide. Psychiatry Res.30;215(3):646-50.

- Atmaca M., Kuloglu M., Tezcan E., Ustundag B., Gecici O., Firidin B.,2002. Serum leptin and cholesterol values in suicide attempters. Neuropsychobiology. 45(3):124-7.

- Atmaca M., Kuloglu M., Tezcan E., Ustundag B.,2003. Serum leptin and cholesterol levels in schizophrenic patients with and without suicide attempts. Acta Psychiatr Scand. 108(3):208-14.

- Atmaca M., Kuloglu M., Tezcan E., Ustundag B.,2008. Serum leptin and cholesterol values in violent and non-violent suicide attempters. Psychiatry Res. 28;158(1):87-91.

- Ayesa-Arriola, R., Canal Rivero, M., Delgado-Alvarado, M., Setien-Suero, E., GonzalezGomez, J., Labad, J., David, A.S., Crespo-Facorro, B., 2018. Low-density lipoprotein cholesterol and suicidal behaviour in a large sample of first-episode psychosis patients. World J. Biol. Psychiatry. 11, 1-4.

- Baek J.H., Kang E.S., Fava, M., Mischoulon, D., Nierenberg, A.A., Yu, B.H., Lee, D., Jeon, H.J., 2014. Serum lipids, recent suicide attempt and recent suicide status in patients with 
major depressive disorder. Prog. Neuropsychopharmacol. Biol. Psychiatry. 51, 113-118.

- Bartoli F., Di Brita C., Crocamo C., Clerici M., Carrà G., 2017a. Lipid profile and suicide attempt in bipolar disorder: A meta-analysis of published and unpublished data. Prog Neuropsychopharmacol. Biol. Psychiatry. 79(Pt B):90-95.

- Bartoli, F., Crocamo, C., Dakanalis A., Riboldi, I., Miotto, A., Brosio, E., Clerici, M., Carrà, G., 2017b. Association between total serum cholesterol and suicide attempts in subjects with major depressive disorder: exploring the role of clinical and biochemical confounding factors. Clin. Biochem.. 50(6):274-278.

- Bradley KA., Case JA., Khan O., Ricart T., Hanna A., Alonso CM., Gabbay V.,2015. The role of the kynurenine pathway in suicidality in adolescent major depressive disorder. Psychiatry Res. 30;227(2-3):206-12.

- Brunner J., Bronisch T., Pfister H., Jacobi F., Höfler M., Wittchen HU., 2006. High cholesterol, triglycerides, and body-mass index in suicide attempters. Arch Suicide Res. 2006;10(1):1-9.

- Cham S., Koslik HJ., Golomb BA.,2016. Mood, Personality, and Behavior Changes During Treatment with Statins: A Case Series. Drug Saf Case Rep Dec;3(1):1.

- Chang SS., Wen CP., Tsai MK., Lawlor DA., Yang YC., Gunnell D.,2011. Adiposity, its related biologic risk factors, and suicide: a cohort study of 542,088 taiwanese adults. Am J Epidemiol. 15;175(8):804-15.

- Chatzittofis A., Nordström P., Hellström C., Arver S., Åsberg M., Jokinen J.,2013. CSF 5HIAA, cortisol and DHEAS levels in suicide attempters. Eur Neuropsychopharmacol. 23(10):1280-7.

- Chapman CL., Mullin K., Ryan CJ., Kuffel A., Nielssen O., Large MM.,2015. Meta-analysis of the association between suicidal ideation and later suicide among patients with either a schizophrenia spectrum psychosis or a mood disorder. Acta Psychiatr Scand. 131(3):162-73. 
- Chokka P., Tancer M., Yeragani VK.,2006. Metabolic syndrome: relevance to antidepressant treatment. . J Psychiatry Neurosci. 31(6):414.

- Conklin SM., Stanford MS.,2007. Premeditated aggression is associated with serum cholesterol in abstinent drug and alcohol dependent men. Psychiatry Res. 15;157(1-3):2837.

- Coryell W., Schlesser M.,2007. Combined biological tests for suicide prediction. Psychiatry Res. 30;150(2):187-91.

- da Graca Cantarelli, M.G., Tramontina, A.C., Leite, M.C., Gonçalves, C.A., 2014. Potential neurochemical links between cholesterol and suicidal behavior. Psychiatry Res. 220(3),745751.

- da Graça Cantarelli M., Nardin P., Buffon A., Eidt MC., Antônio Godoy L., Fernandes BS., Gonçalves CA.,2015. Serum triglycerides, but not cholesterol or leptin, are decreased in suicide attempters with mood disorders. J Affect Disord. 1;172:403-9.

- de Leon, J., Mallory, P., Maw, L., Susce, M.T., Perez-Rodriguez, M.M., Baca-Garcia, E., 2011. Lack of replication of the association of low serum cholesterol and attempted suicide in another country raises more questions. Ann Clin Psychiatry. 23(3), 163-170.

- Diaz-Sastre, C., Baca-Garcia, E., Perez-Rodriguez, M.M., Garcia-Resa, E., Ceverino, A., Saiz-Ruiz, J., Oquendo, M.A., de Leon, J., 2007. Low plasma levels in suicidal males: A gender and body mass index-matched case-control study of suicide attempters and nonattempters. Prog. Neuropsychopharmacol. Biol. Psychiatry. 31, 901-905.

- Elmslie JL., Mann JI., Silverstone JT., Williams SM., Romans SE.,2001. Determinants of overweight and obesity in patients with bipolar disorder. J Clin Psychiatry. 62(6):486-91.

- Fiedorowicz, J.G., Coryell, W.H., 2007. Cholesterol and suicide attempts: a prospective study of depressed inpatients. Psychiatry Res. 152(1), 11-20. 
- Guillem E., Pélissolo A., Notides C, Lépine JP.,2002. Relationship between attempted suicide, serum cholesterol level and novelty seeking in psychiatric in-patients. Psychiatry Res.112(1):83-8.

- Harris CP., von Berg A., Berdel D., Bauer CP., Schikowski T., Koletzko S., Heinrich J., Schulz H., Standl M.,2018, Association of Dietary Fatty Acids with Blood Lipids is Modified by Physical Activity in Adolescents: Results from the GINI plus and LISA Birth Cohort Studies. Nutrients.25;10(10)

- Hibbeln JR., Salem N Jr.,1996. Risks of cholesterol-lowering therapies. Biol Psychiatry. 40(7):686-7. Determining the role of endophenotypes in attempted suicide. Transl. Psychiatry 21;4:e470.

- Jafurulla M., Tiwari S., Chattopadhyay A.,2011. Identification of cholesterol recognition amino acid consensus (CRAC) motif in G-protein coupled receptors. Biochem Biophys Res Commun. 404(1):569-73.

- Jollant F., Lawrence NS., Olie E., O'Daly O., Malafosse A., Courtet P., Phillips ML.,2010. Decreased activation of lateral orbitofrontal cortex during risky choices under uncertainty is associated with disadvantageous decision-making and suicidal behavior. Neuroimage.1; 51(3):1275-81.

- Kim YK., Lee HJ., Kim JY., Yoon DK., Choi SH., Lee MS. 2002. Low serum cholesterol is correlated to suicidality in a Korean sample. Acta Psychiatr Scand.105(2):141-8.

- Kim S.,Lee K-U.,2017. Research on potential biomarker correlates for suicidal behavior: a review. Asia-Pacific Psichiatry;9:e12291

- Kim Y.K., Myint A.M. ,2004. Clinical application of low serum cholesterol as an indicator for suicide risk in major depression. J. Affect. Disord. 81(2):161-6.

- Kunugi H., Takei N., Aoki H., Nanko S.1997. Low serum cholesterol in suicide attempters. Biol Psychiatry.15;41(2):196-200.

- Leppien E., Mulcahy K., Demler TL., Trigoboff E., Opler L.,2018. Effects of Statins and 
Cholesterol on Patient Aggression: Is There a Connection? Innov Clin Neurosci.1;15(34):24-27.

- Lester D.,2002. Serum cholesterol levels and suicide: a meta-analysis. Suicide Life Threat Behav.32(3):333-46.

- Lester D.,2003. Serum cholesterol levels and suicide: a further comment. Suicide Life Threat Behav. 33(3):338-9.

- Loas, G., Dalleau, E., Lecointe, H., Yon, V., 2016. Relationships between anhedonia, alexithymia, impulsivity, suicidal ideation, recent suicide attempt, C-reactive protein and serum lipid levels among 122 inpatients with mood or anxious disorders. Psychiatry Res. 246, 296-302.

- Makris GD., Reutfors J., Ösby U., Isacsson G., Frangakis C., Ekbom A., Papadopoulos FC., 2013. Suicide seasonality and antidepressants: a register-based study in Sweden. Acta Psychiatr Scand. 127(2):117-25

- Marcinko D., Martinac M., Karlović D., Loncar C.,2004. Cholesterol serum levels in violent and non-violent young male schizophrenic suicide attempters. Psychiatr Danub. 16(3):161-4

- McMahon K., Hoertel N., Olfson M., Wall M., Wang S., Blanco C.,2018. Childhood maltreatment and impulsivity as predictors of interpersonal violence, self-injury and suicide attempts: A national study. Psychiatry Res. 18;269:386-393

- Melhem NM., Keilp JG., Porta G., Oquendo MA., Burke A., Stanley B., Cooper TB., Mann JJ., Brent DA.,2016. Blunted HPA Axis Activity in Suicide Attempters Compared to those at High Risk for Suicidal Behavior. Neuropsychopharmacology.41(6):1447-56

- Mensi R., Messaoud A., Mhallah A., Azizi I., Salah WH., Douki W.,Najjar MF., Gaha L.,2016. The association between altered lipid profile and suicide attempt among Tunisian patients with schizophrenia. Ann Gen Psychiatry. 16;15:36.

- Mergl R., Koburger N., Heinrichs K., Székely A., Tóth MD., Coyne J., Quintão S., Arensman E., Coffey C., Maxwell M., Värnik A., van Audenhove C., McDaid D., 
Sarchiapone M., Schmidtke A., Genz A., Gusmão R., Hegerl U.,2015. What Are Reasons for the Large Gender Differences in the Lethality of Suicidal Acts? An Epidemiological Analysis in Four European Countries. PLoS One. 6;10(7):e0129062.

- Messaoud A., Mensi R., Mrad A., Mhalla A., Azizi I., Amemou B., Trabelsi I., Grissa MH., Salem NH., Chadly A., Douki W., Najjar MF., Gaha L.,2017. Is low total cholesterol levels associated with suicide attempt in depressive patients? Ann Gen Psychiatry.17;16:20.

- Moraes JB., Maes M., Roomruangwong C., Bonifacio KL., Barbosa DS., Vargas HO., Anderson G., Kubera M., Carvalho AF., Nunes SOV.,2018. In major affective disorders, early life trauma predict increased nitro-oxidative stress, lipid peroxidation and protein oxidation and recurrence of major affective disorders, suicidal behaviors and a lowered quality of life. Metab Brain Dis. 33(4):1081-1096.

- Mörkl S., Lackner S., Müller W., Gorkiewicz G., Kashofer K., Oberascher A., Painold A., Holl A., Holzer P., Meinitzer A., Mangge H., Holasek S.,2017. Gut microbiota and body composition in anorexia nervosa inpatients in comparison to athletes, overweight, obese, and normal weight controls. Int J Eat Disord.50(12):1421-1431.

- Muldoon MF., Manuck SB., Matthews KA.,1990. Lowering cholesterol concentrations and mortality: a quantitative review of primary prevention trials. BMJ. 11;301(6747):309-14.

- Muldoon MF., Manuck SB., Mendelsohn AB., Kaplan JR., Belle SH.,2001. Cholesterol reduction and non-illness mortality: meta-analysis of randomized clinical trials.BMJ.6;322(7277):11-5

- Nock MK, Kessler RC.,2006. Prevalence of and risk factors for suicide attempts versus suicide gestures: analysis of the National Comorbidity Survey. J Abnorm Psychol 115:61623.

- Nock,M.K., Borges, G., Bromet, E.J., Cha C.B., Kessler R.C., Lee S., 2008.Suicide and suicidal behavior. Epidemiol.Rev. 30(1).133-154.

- Olie E., DingY., Le Bars E., de ChampfleurNM., Mura T., Bonafe A., Courtet P., Jollant F., 
2015. Processing of decision-making and social threat in patients with history of suicidal attempt: a neuroimaging replication study.Psychiatry Res. 234(3):369-77.Papadopolou 2013, da prendere su pube scaricato

- Park, S., Yi K.K., Na, R., Lim, A., Hong, J.P., 2013. No association between serum cholesterol and death by suicide in patients with schizophrenia, bipolar affective disorder, or major depressive disorder. Behav. Brain Funct. 9:45.

- Perera S., Eisen RB., Bhatt M., Dennis BB., Bawor M., El-Sheikh W., DeJesus J., Rangarajan S., Sholer H., Iordan E.,Mackie P., Islam S., Dehghan M., Brasch J.,Meyre D., de Souza R., Thabane L., Samaan Z., 2018. Exploring metabolic factors and health behaviors in relation to suicide attempts: A case-control study. J Affect Disord. 15;229:386395.

- Perez-Rodriguez MM., Baca-Garcia E., Diaz- Sastre C., Garcia-Resa E., Ceverino A., SaizRuiz J., Oquendo MA., de Leon J.,2008. Low serum cholesterol may be associated with suicide attempt history. J Clin Psychiatry 69:1920-7.

- Perroud N., Zewdie S., Stenz L., Adouan W., Bavamian S., Prada P.,Nicastro R., Hasler R., Nallet A., Piguet C., Paoloni-Giacobino A., Aubry JM., Dayer A.,2016. Methylation of serotonin receptor $3 \mathrm{a}$ in $\mathrm{ADHD}$, borderline personality, and bipolar disorders: link with severity of the disorders and childhood maltreatment. Depress Anxiety. 33(1):45-55

- Pompili M., Girardi P., Ruberto A., Tatarelli R.,2005. Suicide in borderline personality disorder: a meta-analysis. Nord J Psychiatry. 59(5):319-24.

- Pompili M., Longo L., Dominici G., Serafini G., Lamis DA., Sarris J.,Amore M., Girardi P.,2017. Polyunsaturated fatty acids and suicide risk in mood disorders: A systematic review. Prog Neuropsychopharmacol Biol Psychiatry. 6;74:43-5

- Rihmer, Z.,Gonda X.,Torzsa P.,Kalabay L., Akiskal HS., Eory A.,2013.Affective temperament,history of suicide attempt and family history of suicide in general practice patients.J.Affect.Disord.149,350-354. 
- Rui H., Qian H., Shi M., Zhang G., Wang L.,2018. Association Between Norepinephrine Transporter Gene rs2242446, rs5569 Polymorphisms and Risk of Major Depressive Disorder. A Meta-analysis. Arch Med Res. 3.

- Ruljancic N., Mihanovic M., Cepelak I.,2011. Thrombocyte serotonin and serum cholesterol concentration in suicidal and non-suicidal depressed patients. Prog Neuropsychopharmacol Biol Psychiatry. 1;35(5):1261-7

- Schreurs, B.G.,2010.The effects of cholesterol on learning and memory. Neuroscience and Biobehavioral Reviews 34,1366-1379.

- Shakeri, J., Farnia, V., Valinia, K., Hashemian, A.H., Bajoghli, H., Holsboer-Trachsler, E., Brand, S., 2015. The relationship between lifetime suicide attempts, serum lipid levels, and metabolic syndrome in patients with bipolar disorders. Int. J. Psychiatry Clin. Pract. 19(2), 124-131.

- Stenbacka, M., Jokinen, J., 2015. Violent and non-violent methods of attempted and completed suicide in Swedish young men: the role of early risk factors. BMC Psychiatry. 15, 196.

- Sudol, K., Mann, J.J., 2017. Biomarkers of Suicide Attempt Behavior: Towards a Biological Model of Risk. Curr. Psychiatry Rep. 19(6), 31.

- Suominen K., Isometsä ÄE., Ostamo A., Lönnqvist J.,2004. Level of suicidal intent predicts overall mortality and suicide after attempted suicide: a 12-year follow-up study. BMC Psychiatry.4:11.

- Tatar ZB., 2018 The relationship between serum lipid levels and lifetime suicide attempts in patients with schizophrenia. Medicine Science. doi: 10.5455/medscience.2018.07.8849

- Tatley M., Savage R.,2007. Psychiatric adverse reactions with statins, fibrates and ezetimibe: implications for the use of lipid-lowering agents. Drug Saf. 30(3):195-201

- Tiao H., Wu S.,2000. Serum cholesterol levels in paranoid and non-paranoid schizophrenia associated with physical violence or suicide attempts in Taiwanese. Psychiatry Res. 
30;96(2):175-8.

- Tripodianakis J., Markianos M., Sarantidis D., Agouridaki M.,2002. Biogenic amine turnover and serum cholesterol in suicide attempt. Eur Arch Psychiatry Clin Neurosci. 252(1):38-43.

- Troisi A., 2011. Low cholesterol is a risk factor for attentional impulsivity in patients with mood symptoms. Psychiatry Res. 188(1), 83-87.

- Vargas HO., Nunes SO., Pizzo de Castro M., Bortolasci CC., Sabbatini Barbosa D., Kaminami Morimoto H., Venugopal K., Dodd S., Maes M., Berk M.,2013. Oxidative stress and lowered total antioxidant status are associated with a history of suicide attempts. J AffectDisord.25;150(3):923-30.

- Volpato S., Zuliani G, Guralnik JM, Palmieri E, Fellin R.,2001. The inverse association between age and cholesterol level among older patients: the role of poor health status. Gerontology.47(1):36-45.

- Von Elm, E., Altman, D.G., Egger, M., Pocock, S.J., Gotzsche, P.C., Vandenbroucke, J.P.; Iniciativa STROBE 2008. [The Strengthening the Reporting of Observational Studies in Epidemiology (STROBE) statement: guidelines for reporting observational studies]. Rev. Esp. Salud Publica. 82(3), 251-259.

- Voulalas PJ., Schetz J., Undieh A.S.,2011.Differential subcellular distribution of rat brain dopamine receptor sand subtype-specific redistribution induced by cocaine. Molecular and CellularNeurosciences 46,645-654.

- Wallner B., Machatschke IH.,2009. The evolution of violence in men: the function of central cholesterol and serotonin. Prog Neuropsychopharmacol Biol Psychiatry. $30 ; 33(3): 391-7$

- Wu, S., Ding, Y., Wu, F., Xie, G., Hou, J., Mao, P., 2016. Serum lipid levels and suicidality: a meta-analysis of 65 epidemiological studies. J Psychiatry Neurosci. 41(1), 56-69.

- Yang CC., Jick SS, Jick H., 2003. Lipid-lowering drugs and the risk of depression and 
suicidal behavior. Arch Intern Med. 8;163(16):1926-33

- Zhang J., McKeown RE., Hussey JR., Thompson SJ., Woods JR., Ainsworth BE.,2005. Low HDL cholesterol is associated with suicide attempt among young healthy women: the Third National Health and Nutrition Examination Survey. J Affect Disord. 89(1-3):25-33 